\title{
Reverse mathematics and the equivalence of definitions for well and better quasi-orders
}

\author{
Peter Cholak \\ Department of Mathematics, University of Notre Dame, USA \\ Alberto Marcone \\ Dipartimento di Matematica e Informatica, Università di Udine, Italy \\ Reed Solomon \\ Department of Mathematics, University of Connecticut, USA
}

August 28, 2003

\section{Introduction}

In reverse mathematics, one formalizes theorems of ordinary mathematics in second order arithmetic and attempts to discover which set theoretic axioms are required to prove these theorems. Often, this project involves making choices between classically equivalent definitions for the relevant mathematical concepts. In this paper, we consider a number of equivalent definitions for the notions of well quasi-order and better quasi-order and examine how difficult it is to prove the equivalences of these definitions.

As usual in reverse mathematics, we work in the context of subsystems of second order arithmetic and take $\mathrm{RCA}_{0}$ as our base system. $\mathrm{RCA}_{0}$ is the subsystem formed by restricting the comprehension scheme in second order arithmetic to $\Delta_{1}^{0}$ formulas and adding a formula induction scheme for $\boldsymbol{\Sigma}_{1}^{0}$ formulas. For the purposes of this paper, we will be concerned with fairly weak extensions of $\mathrm{RCA}_{0}$ (indeed strictly weaker than the subsystem $\mathrm{ACA}_{0}$ which is formed by extending the comprehension scheme in $\mathrm{RCA}_{0}$ to cover all arithmetic formulas) obtained by adjoining certain combinatorial principles to $\mathrm{RCA}_{0}$. Among these, the most widely used in reverse mathematics is Weak König's Lemma; the resulting theory $\mathrm{WKL}_{0}$ is extensively documented in [11] and elsewhere.

We give three other combinatorial principles which we use in this paper. In these principles, we use $k$ to denote not only a natural number but also the finite set $\{0, \ldots, k-1\}$. For any

Cholak's research was partially supported by NSF Grants DMS 99-88716 and DMS 02-45167. Marcone's research was partially supported by INdAM of Italy and he thanks the Math Department of the University of Notre Dame for its kind hospitality. Solomon's research was partially supported by an NSF Postdoctoral Fellowship. 


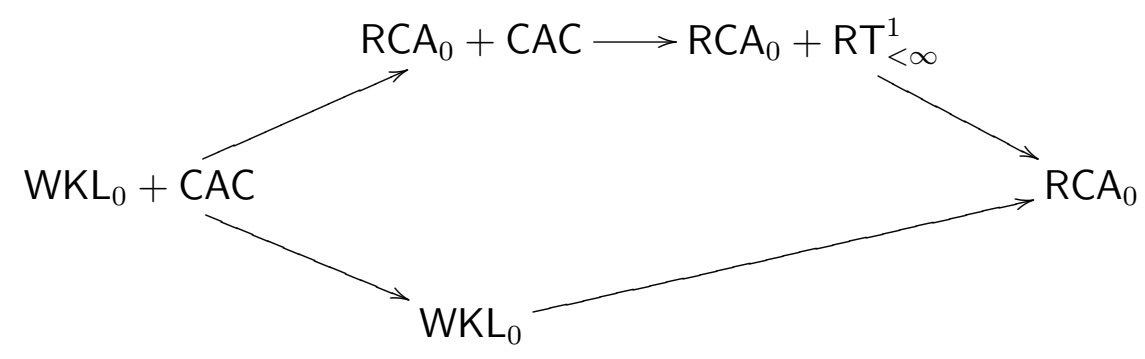

Diagram 1: none of the missing arrows hold except possibly the arrow from $\mathrm{RCA}_{0}+\mathrm{CAC}$ to $\mathrm{WKL}_{0}+\mathrm{CAC}$.

set $X$ and any $n \in \mathbb{N}$, we let $[X]^{n}$ denote the set of all subsets of $X$ of size $n$. Similarly, $[X]^{<\omega}$ denotes the set of all finite subsets of $X$ and $Y \in[X]^{\omega}$ is an abbreviation for the statement that $Y$ is an infinite subset of $X$. The Pigeonhole principle is the statement

$$
\forall k \forall f: \mathbb{N} \rightarrow k \exists A \in[\mathbb{N}]^{\omega} \exists i<k \forall j \in A(f(j)=i)
$$

and is denoted $\mathrm{RT}_{<\infty}^{1}$. (This notation comes from thinking of the principle as a version of Ramsey's Theorem for singletons and finitely many colors.) Ramsey's Theorem for pairs and two colors is the statement

$$
\forall f:[\mathbb{N}]^{2} \rightarrow 2 \exists A \in[\mathbb{N}]^{\omega} \exists i<2 \forall j \in[A]^{2}(f(j)=i)
$$

and is denoted $\mathrm{RT}_{2}^{2}$. The chain-antichain principle is denoted CAC and says that every infinite partial order has either an infinite chain (that is, an infinite linearly ordered subset) or an infinite antichain.

It is known that none of these principles is provable in $\mathrm{WKL}_{0}$ and that over $\mathrm{RCA}, \mathrm{RT}_{2}^{2}$ implies CAC which implies $\mathrm{RT}_{<\infty}^{1} . \mathrm{RT}_{<\infty}^{1}$ is the only one of these principles which is true in REC, the $\omega$-model of the computable sets, and therefore, the implication from CAC to $\mathrm{RT}_{<\infty}^{1}$ cannot be reversed. It is an open question in reverse mathematics whether the implication from $\mathrm{RT}_{2}^{2}$ to $\mathrm{CAC}$ can be reversed, even over $\mathrm{WKL}_{0}$. Diagram 1 illustrates the relationship of these principles below the system $\mathrm{WKL}_{0}+\mathrm{CAC}$. (For more information on reverse mathematics and Ramsey's Theorem, see Cholak, Jockusch and Slaman [1].)

In Section 2, we give some background on well quasi-orders including five classically equivalent definitions for this concept. We show exactly how these equivalences are related in each of the systems presented in Diagram 1 as well as in the $\omega$ model REC, with the exception of one potential implication in the system $\mathrm{RCA}_{0}+\mathrm{CAC}$. (This potentially missing implication depends on the open question of determining whether $R C A_{0}+C A C$ implies $W_{K L}+C A C$.) Our main results are summarized in Diagrams 2, 3 and 4 and in Table 2. The proofs of these results are given in Section 3. Many of our results show that some implication between these equivalent definitions is not provable in $\mathrm{RCA}_{0}$ by producing an appropriate "computable counterexample" to the classical equivalence. In some cases, these counterexamples can be extended to show that the implication is not even provable in $\mathrm{WKL}_{0}$. However, they do not lead to a reversal to a system stronger than $\mathrm{RCA}_{0}$ in the usual reverse mathematics style. 
Therefore, although we can describe the exact relationships between the definitions in most of the systems above, we fall short of the ideal goal of giving an exact classification of the proof-theoretic strength for most of these equivalences.

In Section 4, we take a break from studying the reverse mathematics of equivalent definitions for well quasi-orders to look at the difficulty of proving that the notion of well quasi-order is closed under products and intersections. Of course, we can check the difficulty of proving the closure properties for each of our five definitions for a well quasi-order. It is known that for any of these definitions, $\mathrm{RCA}_{0}$ is strong enough to prove that the closure under either the operation of intersection or product implies the closure under the other operation. For the strongest definition of well quasi-order, $\mathrm{RCA}_{0}$ suffices to prove the closure under these operation, but for the other four definitions, we show that there is an $\omega$-model of $\mathrm{WKL}_{0}$ which is not closed under either of these operations. Furthermore, we can mix-and-match these definitions by asking how difficult it is to prove that if we start with a well quasi-order under one definition, then the intersection or product satisfies another one of the definitions. We classify exactly which combinations are provable in $\mathrm{RCA}_{0}$ and which combinations are not provable in $\mathrm{WKL}_{0}$.

In Section 5, we return to studying the reverse mathematics of equivalent definitions by considering the notion of a better quasi-orders. We present some background on better quasiorders and examine the relationship between the definition of better quasi-order in terms of blocks and the definition in terms of barriers. Because it is shown in [7] that the closure of better quasi-orders under products and intersections implies ACA $_{0}$ (which is a strictly stronger subsystem than any mentioned in Diagram 1), we leave the project of classifying the proof-theoretic complexity of these operations as an open problem.

For a general survey of the classical theory of well quasi-orders and better quasi-orders, including important examples and more equivalent definitions, the reader is referred to Milner [8] and Simpson [9]. For more background in reverse mathematics, the reader is referred to Simpson [11]. For more on wqo and bqo theory from the reverse mathematics point of view, the reader is referred to Marcone [7].

Before beginning the mathematical part of the paper, we fix some notation for sequences. Given any set $A, \mathrm{RCA}_{0}$ is strong enough to form $A^{<\omega}$, the set of all finite sequences of elements of $A$. If $a_{1}, \ldots, a_{n} \in A$, then we write the sequence with these elements as $\left\langle a_{1}, \ldots, a_{n}\right\rangle$. If $s, t \in A^{<\omega}$, then $|s|$ denotes the length of $s$ and $s * t$ denotes the concatenation of $s$ and $t$. If $a \in A$, we sometimes write $s * a$ in place of $s *\langle a\rangle$. We write $s \sqsubseteq t$ to denote that $s$ is an initial segment of $t$ and $s \sqsubset t$ to denote that $s$ is a proper initial segment of $t$. Finally, if $n<|s|$, then the restriction of $s$ to $n$, written $s \mid n$, is the initial segment of $s$ of length $n$.

\section{Well quasi-orders}

We begin with the basic definitions associated with quasi-orders and well quasi-orders.

Definition 2.1. $\left(\mathrm{RCA}_{0}\right)$ A quasi-order is a pair $(Q, \preceq)$ such that $Q$ is a set and $\preceq$ is a transitive reflexive relation on $Q$.

Partial orders are the simplest examples of quasi-orders as a partial order is a quasi-order 
which also satisfies antisymmetry. We can transform a quasi-order $Q$ into a partial order using the equivalence relation defined by $n \sim m$ if and only if $n \preceq m$ and $m \preceq n$. The quotient structure $Q / \sim$ is a partial order which can be formed using $\Delta_{1}^{0}$ comprehension in $\mathrm{RCA}_{0}$. Much of the standard terminology and notation for partial orders is used in the context of quasi-orders. For example, we write $i \perp j$ to indicate that $i$ and $j$ are incomparable under $\preceq$ and we write $x \prec y$ if $x \preceq y$ and $y \npreceq x$. Furthermore, we sometimes use $\leq_{\mathbb{N}}$ to denote the order relation given by the symbol $\leq$ in the language of second order arithmetic. This notation helps to emphasize when we are comparing elements of a quasi-order via the quasiorder relation and when we are comparing them via the underlying structure of arithmetic. We use this notation when the distinction between these orders is not immediately clear from the context.

$A \subseteq Q$ is an antichain if for all $i \neq j \in A, i \perp j$. An infinite ascending chain in $Q$ is a function $f: \mathbb{N} \rightarrow Q$ such that for all $i<_{\mathbb{N}} j, f(i) \prec f(j)$. Similarly, a function $f$ is an infinite descending chain in $Q$ if for all $i<_{\mathbb{N}} j, f(j) \prec f(i)$. Any antichain in $Q$ gives rise to a corresponding antichain in $Q / \sim$ and vice versa. Infinite ascending chains and infinite descending chains in $Q$ and $Q / \sim$ are similarly related. Therefore, we frequently work with partial orders rather than quasi-orders.

A quasi-order $(Q, \preceq)$ is called linear if for all $i, j \in Q$, either $i \preceq j$ or $j \preceq i$. For a linear quasi-order $Q, Q / \sim$ is a linear order. If $\preceq$ is a quasi-order on $Q$ and ${ }_{L}$ is a linear quasi-order on $Q$, then we say $\preceq_{L}$ is a linear extension of $\preceq$ if for all $i, j \in Q, i \preceq j$ implies $i \preceq L j$ and $i \sim_{L} j$ implies $i \sim j$. Notice that these conditions imply that $i \sim j$ if and only if $i \sim_{L} j$. Therefore, the linear extensions of a quasi-order $Q$ correspond exactly to the linear extensions of the partial order $Q / \sim$. A linear quasi-order $\preceq_{L}$ on $Q$ is said to be well ordered if the induced linear order on $Q / \sim_{L}$ is a well order. (In $\mathrm{RCA}_{0}$, the definition of a well order stating that each nonempty subset has a least element and the definition stating that there are no infinite descending chains are equivalent. We will use the definition in terms of no infinite descending chains.)

Definition 2.2. $\left(\mathrm{RCA}_{0}\right)$ A quasi-order $(Q, \preceq)$ is a well quasi-order (or wqo) if for every function $f: \mathbb{N} \rightarrow Q$ there exist $m<_{\mathbb{N}} n$ such that $f(m) \preceq f(n)$.

As we shall see, there are many classically equivalent definitions for a well quasi-order, but the definition above is frequently used as the "official" definition for a well quasi-order in reverse mathematics. The simplest examples of well quasi-orders are well founded partial orders which have only finite antichains. For example, the $m$-fold cartesian product $\mathbb{N}^{m}$ ordered by

$$
\left\langle a_{0}, \ldots, a_{m-1}\right\rangle \leq_{\mathbb{N}^{m}}\left\langle b_{0}, \ldots, b_{m-1}\right\rangle \Leftrightarrow \forall i<m\left(a_{i} \leq b_{i}\right)
$$

is a well quasi-order.

One natural question to ask about well quasi-orders is how difficult it is to prove that a given wqo is in fact well quasi-ordered. Simpson [10] showed that (given a suitable set of notations for the ordinals below $\epsilon_{0}$ ) the system $\mathrm{RCA}_{0}$ can prove that for all $m \in \mathbb{N}$, the statement that $\mathbb{N}^{m}$ (ordered as above) is a wqo is equivalent to the statement that $\omega^{m}$ is a well order. It follows that for each standard $m, \mathrm{RCA}_{0}$ can prove that $\mathbb{N}^{m}$ is a well quasi-order, but that $\mathrm{RCA}_{0}$ cannot prove the uniform statement that for all $m, \mathbb{N}^{m}$ is a wqo. 


\begin{tabular}{|c|c|c|c|c|}
\hline$\rightarrow$ & wqo & wqo(set) & wqo(anti) & wqo(ext) \\
\hline wqo & $\mathrm{RCA}_{0} \vdash$ & $\begin{array}{l}\rightarrow \mathrm{RT}_{<\infty}^{1} \\
\mathrm{WKL}_{0} \forall \\
(*)\end{array}$ & $\mathrm{RCA}_{0} \vdash$ & \\
\hline wqo(set) & $\mathrm{RCA}_{0} \vdash$ & $\mathrm{RCA}_{0} \vdash$ & $\mathrm{RCA}_{0} \vdash$ & \\
\hline wqo(anti) & & $\begin{array}{l}\rightarrow \mathrm{RT}_{<\infty}^{1} \\
\mathrm{WKL}_{0}\end{array}$ & $\mathrm{RCA}_{0} \vdash$ & \\
\hline wqo(ext) & & & & $\mathrm{RCA}_{0} \vdash$ \\
\hline
\end{tabular}

Table 1: Previously known results

Rather than pursue the task of describing the quasi-orders which are well quasi-orders in $\mathrm{RCA}_{0}$, we will look at various classically equivalent definitions for the notion of a well quasiorder and examine how difficult it is to prove their equivalence. An important notion for this direction of study is the finite basis property.

Definition 2.3. $\left(\mathrm{RCA}_{0}\right)$ A quasi-order $\preceq$ on $Q$ has the finite basis property if for every $X \subseteq Q$ there exists a finite $F \subseteq X$ such that $\forall x \in X \exists y \in F(y \preceq x)$.

Theorem 2.4 lists several classically equivalent definitions for a well quasi-order. Each of these equivalences can be proved using either Ramsey's Theorem for pairs or Weak König's Lemma, and the proofs can be formalized in $\mathrm{WKL}_{0}+\mathrm{RT}_{2}^{2}$.

Theorem 2.4. For any quasi-order $(Q, \preceq)$, the following are equivalent.

1. $(Q, \preceq)$ is a well quasi-order.

2. For all $f: \mathbb{N} \rightarrow Q$ there is an infinite set $A$ such that for all $i, j \in A, i<j \rightarrow f(i) \preceq$ $f(j)$.

3. $(Q, \preceq)$ has no infinite descending chains and no infinite antichains.

4. Every linear extension of $(Q, \preceq)$ is well ordered.

5. $(Q, \preceq)$ has the finite basis property.

Our goal is to examine how difficult it is to prove these equivalences from the point of view of reverse mathematics. We use wqo to denote our official definition of well quasi-order in $\mathrm{RCA}_{0}$. We let wqo(set) denote the condition in 2 , wqo(anti) denote the condition in 3 , wqo(ext) denote the condition in 4 , and wqo(fbp) denote the condition in 5 . That is, we say $Q$ satisfies wqo(ext) to mean that every linear extension of $Q$ is well ordered, and we write wqo(ext) $\rightarrow$ wqo(anti) to denote the statement: for all quasi-orders $Q$, if every linear extension of $Q$ is well ordered, then $Q$ has no infinite descending chains and no infinite antichains.

A number of facts about the relationships between these notions are already known. For example, it follows trivially from the definitions that $\mathrm{RCA}_{0} \vdash$ wqo(set) $\rightarrow$ wqo. The following theorem summarizes the results of Lemmas 4.2, 4.4 and 4.8 in Marcone [7] and Lemma 3.2 in 
Simpson [10]. (Lemma 3.2 in [10] is stated in terms of partial orders rather than quasi-orders, but the proof translates quite easily.)

\section{Theorem 2.5 (Marcone, Simpson). $\quad$ 1. $\mathrm{RCA}_{0} \vdash w q o \rightarrow w q o($ anti).}

2. $\mathrm{RCA}_{0} \vdash(w q o \rightarrow w q o($ set $)) \rightarrow \mathrm{RT}_{<\infty}^{1}$.

3. $\mathrm{RCA}_{0} \vdash w q o \leftrightarrow w q o(f b p)$.

Because $\mathrm{WKL}_{0} \not \forall \mathrm{RT}_{<\infty}^{1}$, statement 2 in Theorem 2.5 implies that $\mathrm{WKL}_{0} \not$ wqo $\rightarrow$ wqo(set). We can draw a number of other simple consequences from these results. For example, since $\mathrm{RCA}_{0} \vdash$ wqo(set) $\rightarrow$ wqo and $\mathrm{RCA}_{0} \vdash$ wqo $\rightarrow$ wqo(anti), we have $\mathrm{RCA}_{0} \vdash \mathrm{wqo}($ set $) \rightarrow$ wqo(anti). For a second example, fix any model $\mathcal{M}$ of $\mathrm{RCA}_{0}$ and wqo(anti) $\rightarrow$ wqo(set). Since $\mathrm{RCA}_{0} \vdash$ wqo $\rightarrow$ wqo(anti), we have that $\mathcal{M} \models$ wqo $\rightarrow$ wqo(set) and hence $\mathcal{M} \models \mathrm{RT}_{<\infty}^{1}$. Therefore, $\mathrm{RCA}_{0} \vdash($ wqo(anti) $\rightarrow$ wqo(set) $) \rightarrow \mathrm{RT}_{<\infty}^{1}$. We summarize the results in Theorem 2.5 and their simple consequences in Table 1. To read this table, consider the entries in square marked $(*)$. The $\rightarrow \mathrm{RT}_{<\infty}^{1}$ indicates that (over $\mathrm{RCA}_{0}$ ) wqo $\rightarrow$ wqo(set) implies $\mathrm{RT}_{<\infty}^{1}$ and the $\mathrm{WKL}_{0} \nvdash$ indicates that $\mathrm{WKL}_{0} \nvdash$ wqo $\rightarrow$ wqo(set). In Table 1, we did not put a row or column for the condition wqo(fbp) because by statement 3 in Theorem 2.5, the row or column for wqo(fbp) would be equivalent to the corresponding row or column for wqo.

Notice that while statement 2 in Theorem 2.5 implies that $\mathrm{WKL}_{0} \not \forall$ wqo $\rightarrow$ wqo(set), it does not rule out the possibility that this implication is true in all $\omega$-models of $\mathrm{RCA}_{0}$. In particular, it leaves open the question of whether REC, the $\omega$-model consisting of the computable sets, is a model for this implication.

In Section 3, we improve the results in Theorem 2.5 to give all the results contained in Table 2. This table is read exactly as Table 1 and the information which is not contained in Table 1 will be proved in Section 3. As a reference to the reader, for all the results in Table 2 which do not appear in Table 1, we indicate in parentheses the number of the theorem, lemma or corollary in which we prove the particular statement.

The reverse mathematics content of Table 2 can be more easily visualized and summarized as follows. The system $\mathrm{WKL}_{0}+\mathrm{CAC}$ is strong enough to prove the equivalence between all of the definitions for well quasi-order considered in this paper. Over RCA $\mathrm{A}_{0}$, the only implications between the various definitions are those shown in Diagram 2 plus the fact that $\mathrm{RCA}_{0} \vdash$ wqo $\leftrightarrow \mathrm{wqo}(\mathrm{fbp})$. Because we give computable counterexamples to implications which do not hold and because REC is a model for $\mathrm{RCA}_{0}+\mathrm{RT}_{<\infty}^{1}$, Diagram 2 also illustrates which implications hold in the $\omega$-model REC and in the subsystem $\mathrm{RCA}_{0}+\mathrm{RT}_{<\infty}^{1}$. Over $\mathrm{WKL}_{0}$, the implications which hold are exactly those shown in Diagram 3.

Over RCA + CAC, the known implications are those shown in Diagram 4. Unlike Diagrams 2 and 3 , it is possible that the lone missing implication (from wqo(ext) the other three conditions) in Diagram 4 does hold. However, notice that since $\mathrm{WKL}_{0} \vdash$ wqo(ext) $\rightarrow$ wqo, any proof that $\mathrm{RCA}_{0}+\mathrm{CAC} \forall \forall$ wqo $($ ext $) \rightarrow$ wqo would yield a proof that $\mathrm{RCA}_{0}+\mathrm{CAC} \forall \mathrm{WKL}_{0}$ and hence $R C A_{0}+R_{2}^{2} \not W K L_{0}$. Such a proof would answer one of the major open questions in reverse mathematics. 


\begin{tabular}{|c|c|c|c|c|}
\hline$\rightarrow$ & wqo & wqo(set) & wqo(anti) & wqo(ext) \\
\hline wqo & $\mathrm{RCA}_{0} \vdash$ & $\begin{array}{l}\rightarrow \mathrm{RT}_{<\infty}^{1} \\
\mathrm{WKL}_{0} \forall \\
\mathrm{CAC} \vdash \\
(3.4) \\
\mathrm{REC} \forall \forall \\
(3.7)\end{array}$ & $\mathrm{RCA}_{0} \vdash$ & $\begin{array}{l}\mathrm{RCA}_{0} \vdash \\
(3.12)\end{array}$ \\
\hline wqo(set) & $\mathrm{RCA}_{0} \vdash$ & $\mathrm{RCA}_{0} \vdash$ & $\mathrm{RCA}_{0} \vdash$ & $\begin{array}{l}\mathrm{RCA}_{0} \vdash \\
(3.13)\end{array}$ \\
\hline wqo(anti) & $\begin{array}{l}\mathrm{REC} \forall \\
(3.9) \\
\mathrm{CAC} \vdash \\
(3.5) \\
\mathrm{WKL}_{0} \forall \\
(3.11)\end{array}$ & $\begin{array}{l}\leftrightarrow \text { CAC } \\
(3.3) \\
\mathrm{WKL}_{0} \forall \\
(3.6)\end{array}$ & $\mathrm{RCA}_{0} \vdash$ & $\begin{array}{l}\mathrm{REC} \not \\
(3.10) \\
\mathrm{CAC} \vdash \\
(3.5) \\
\mathrm{WKL}_{0} \forall \\
(3.19)\end{array}$ \\
\hline wqo(ext) & $\begin{array}{l}\text { REC } \not \models \\
(3.21) \\
\text { WKL }_{0} \vdash \\
(3.17)\end{array}$ & $\begin{array}{l}\rightarrow \mathrm{RT}_{<\infty}^{1} \\
(3.20) \\
\mathrm{WKL}_{0} \forall \\
(3.20) \\
\mathrm{REC} \not \models \\
(3.24) \\
\mathrm{WKL}_{0}+\mathrm{CAC} \vdash \\
(3.4+3.17)\end{array}$ & $\begin{array}{l}\mathrm{REC} \not \models \\
(3.23) \\
\mathrm{WKL}_{0} \vdash \\
(3.18)\end{array}$ & $\mathrm{RCA}_{0} \vdash$ \\
\hline
\end{tabular}

Table 2: Equivalent definitions for well quasi-orders

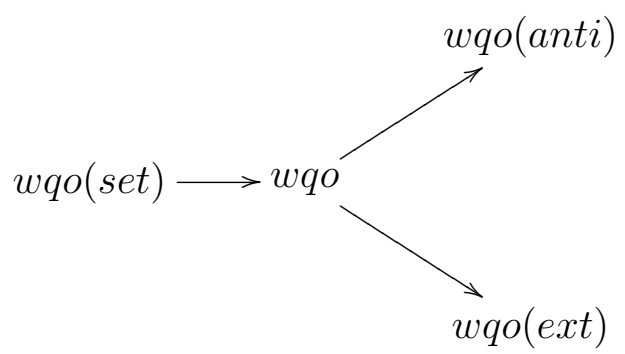

Diagram 2: the exact implications in $R C A_{0}$, in REC and in $\mathrm{RCA}_{0}+\mathrm{RT}_{<\infty}^{1}$. 


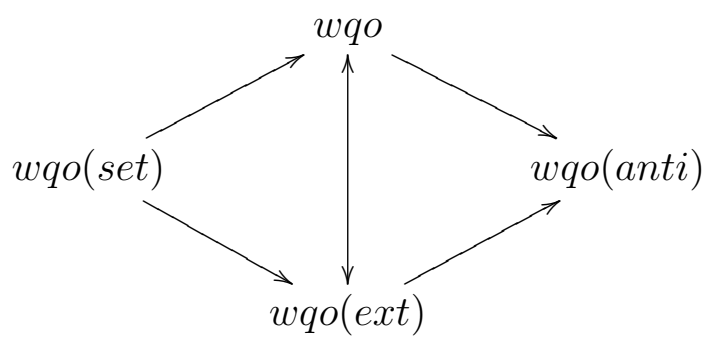

Diagram 3: the exact implications in $\mathrm{WKL}_{0}$.

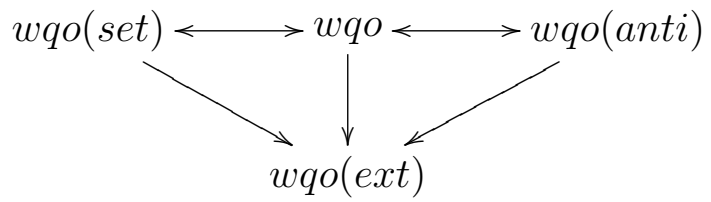

Diagram 4: the implications in $\mathrm{RCA}_{0}+\mathrm{CAC}$. It remains open whether the missing arrows hold.

Finally, we note that there are many other combinatorial principles which sit below $\mathrm{WKL}_{0}+$ $\mathrm{RT}_{2}^{2}$ in reverse mathematics and which could be compared to the implications examined in this paper. For example, Hirschfeldt and Shore [5] have examined variations on the statement that every infinite linear order has either an infinite ascending sequence or an infinite descending sequence (denote ASDS). It is easy to show that RCA $\mathrm{A}_{0}$ suffices to prove that wqo $\rightarrow$ wqo(set) implies ASDS. To see this claim, we assume that wqo $\rightarrow$ wqo(set) and we work in RCA $_{0}$. Fix a linear order $L$ and assume that $L$ has no infinite descending sequences. Then $L$ obviously satisfies wqo. Let $f$ be a one-to-one enumeration of $L$ and let $A$ be the infinite set obtained from the statement of wqo(set). For any $i, j \in A$, if $i<_{\mathbb{N}} j$, then $f(i)<_{L} f(j)$ and so $A$ gives us the ascending chain required for ASDS. It follows from the work in [5] that $\mathrm{COH} \forall$ wqo $\rightarrow$ wqo(set) where $\mathrm{COH}$ is the combinatorial statement that every sequence of sets $X_{i}, i \in \mathbb{N}$, has an infinite cohesive set $C$. That is, for all $i$, either $C$ is contained in $X_{i}$ except for finitely many elements or $X_{i}$ is contained in $C$ for finitely many elements. We will not explore these connections any further in this paper, but we wanted to alert the reader that the situation concerning combinatorial principals below $\mathrm{RT}_{2}^{2}$ is diverse and complicated.

\section{Proofs for well quasi-order results}

We begin this section by showing that we can replace the use of $\mathrm{RT}_{2}^{2}$ by CAC in the proofs of several of our implications. Since it is not known whether CAC implies $\mathrm{RT}_{2}^{2}$, it is possible that this replacement is not actually an improvement on the known results. First, we show two consequences of $\mathrm{RCA}_{0}+\mathrm{CAC}$. (We assume that Lemma 3.1 is already widely known and Lemma 3.2 is due to Hirschfeldt and Shore [5]. We include proofs only for the sake of completeness.) 
Lemma 3.1. $\mathrm{RCA}_{0}+\mathrm{CAC} \vdash \mathrm{RT}_{<\infty}^{1}$.

Proof. Fix $k \in \mathbb{N}$ and let $f: \mathbb{N} \rightarrow k$. We need to find an infinite set on which $f$ is constant. Define a partial order $<_{f}$ on $\mathbb{N}$ by $n<_{f} m$ if and only if $n<_{\mathbb{N}} m$ and $f(n)=f(m)$. It is clear that for any $n$ and $m$, if $f(n)=f(m)$, then either $n \leq_{f} m$ or $m \leq_{f} n$. Therefore, any antichain in our partial order has size at most $k$. Applying CAC, we obtain an infinite chain in this partial order. However, $f$ must be constant on any chain so we have our desired infinite set.

Lemma 3.2. $\mathrm{RCA}_{0}+\mathrm{CAC}$ suffices to prove that for every partial order $P$ and for every infinite chain $C \subset P$, either $C$ contains an infinite ascending sequence or $C$ contains an infinite descending sequence.

Proof. Fix a partial order $P$ and an infinite chain $C \subseteq P$. We define a new partial order $\leq^{\prime}$ on $C$ by setting $n \leq^{\prime} m$ if and only if $n \leq_{P} m$ and $n \leq_{\mathbb{N}} m$. Applying CAC, there is either an infinite chain or an infinite antichain in $\leq^{\prime}$. It is not hard to check that RCA $_{0}$ suffices to prove that an infinite chain in $\leq^{\prime}$ corresponds to an infinite increasing sequence in $\leq_{P}$ and an infinite antichain in $\leq^{\prime}$ corresponds to an infinite descending sequence in $\leq_{P}$. (To see these correspondences, just list the chain or antichain in $\leq_{\mathbb{N}}$-increasing order.)

Lemma 3.3. $\left(\mathrm{RCA}_{0}\right)$ The following are equivalent:

\section{CAC}

2. $w q o($ anti $) \rightarrow w q o($ set $)$

Proof. We first show that CAC is strong enough to prove wqo(anti) $\rightarrow$ wqo(set). Fix any quasi-order $(Q, \prec)$ which has no infinite descending chains or infinite antichains and fix any function $f: \mathbb{N} \rightarrow Q$. Consider the equivalence relation $\sim$ on $Q$ defined by $x \sim y$ if and only if $x \preceq y$ and $y \preceq x$. If the range of $f$ intersects only finitely many equivalence classes under $\sim$, then we can apply $\mathrm{RT}_{<\infty}^{1}$ to obtain an infinite set $A$ such that $f$ restricted to $A$ maps into a single equivalence class of $\sim$. For any $i, j \in A$, we have $f(i) \preceq f(j)$, so $A$ satisfies wqo(set) for the map $f$. Therefore, by Lemma 3.1, we can assume that $f$ maps into infinitely many equivalence classes under $\sim$.

We now look at the partial order $Q / \sim$ and we view $f$ as mapping into this partial order. By our assumption the range of $f$ when viewed as mapping into $Q / \sim$ is infinite. We abuse notation slightly and write $x \preceq y$ to denote the order relation in $Q / \sim$. We can assume without loss of generality that $f$ is one-to-one, since $f$ can be replaced by a one-to-one function $h$ with the same range and such that for all $n$ and $k$, if $h(n)=k$, then there is an $m \geq n$ such that $f(m)=k$. We need to find an infinite set $A$ such that for all $i, j \in A$, if $i<j$, then $f(i) \preceq f(j)$. We define an auxiliary partial order $P$ with domain $\mathbb{N}$ such that $n<_{P} m$ if and only if $f(n) \prec f(m)$. Notice that any antichain in $\leq_{P}$ corresponds to an antichain in $Q / \sim$, which in turn corresponds to an antichain in $Q$. Since there are no infinite antichains in $Q$, there cannot be an infinite antichain in $P$.

Since $P$ is an infinite partial order, we can apply CAC to it. Furthermore, since $P$ has no infinite antichains, we must obtain an infinite chain when we do this. However, by Lemma 
3.2, CAC can prove that this infinite chain contains either an infinite descending sequence or an infinite ascending sequence. Since an infinite descending sequence in $P$ would yield an infinite descending sequence in $Q$, there cannot be such a sequence. Therefore, we obtain an infinite ascending sequence $b_{0}<_{P} b_{1}<_{P} \cdots$ in $P$. We define an infinite set $A$ by enumerating it in increasing order $a_{0}<_{\mathbb{N}} a_{1}<_{\mathbb{N}} \cdots$ as follows. Set $a_{0}=b_{0}$. If $a_{i}=b_{j}$, then let $a_{i+1}=b_{k}$ where $k$ is the least index such that $k>j$ and $b_{k}>_{\mathbb{N}} b_{j}$. Notice that $a_{i}<_{\mathbb{N}} a_{j}$ if and only if $i<_{\mathbb{N}} j$ and that $a_{i}<_{\mathbb{N}} a_{j}$ implies $a_{i}<_{P} a_{j}$ which implies $f\left(a_{i}\right) \prec f\left(a_{j}\right)$. Therefore, $A$ is the desired set for the function $f$ in the statement of wqo(set).

We next show that wqo(anti) $\rightarrow$ wqo(set) implies CAC. Fix an infinite partial order $P$. We need to show that $P$ has either an infinite chain or an infinite antichain. Assume that $P$ does not have an infinite descending chain or an infinite antichain. Then $P$ satisfies wqo(anti) and by wqo(anti) $\rightarrow$ wqo(set) we have that $P$ satisfies wqo(set). Let $f$ be a one-to-one map from $\mathbb{N}$ onto $P$. The infinite set $A$ from the statement of wqo(set) gives the desired infinite chain.

Corollary 3.4. $\mathrm{RCA}_{0}+\mathrm{CAC} \vdash w q o \rightarrow$ wqo(set).

Proof. This corollary follows from Lemma 3.3 since $\mathrm{RCA}_{0} \vdash$ wqo $\rightarrow$ wqo(anti) (from Theorem 2.5).

Corollary 3.5. $\mathrm{RCA}_{0}+\mathrm{CAC}$ suffices to prove wqo(anti) $\rightarrow$ wqo and wqo(anti) $\rightarrow$ wqo(ext).

Proof. These two statements follow from Lemma 3.3 plus $\mathrm{RCA}_{0} \vdash$ wqo(set) $\rightarrow$ wqo (trivial from the definitions) and $\mathrm{RCA}_{0} \vdash$ wqo(set) $\rightarrow$ wqo(ext) (to be proved in Lemma 3.12).

Corollary 3.6. $\mathrm{WKL}_{0} \nvdash$ wqo (anti) $\rightarrow$ wqo(set)

Proof. This corollary follows from Lemma 3.3 because $\mathrm{WKL}_{0} \not \forall C A C$.

Next we show that several of the implications between the classical definitions for a well quasi-order are not true in REC. Recall that REC is the $\omega$ model consisting of the computable sets and that it is the minimal $\omega$ model of $R C A_{0}$.

Lemma 3.7. REC $\not w q o \rightarrow w q o($ set $)$.

Proof. Let $L$ be a computable linear order which has order type $\omega+\omega^{*}$ but which does not have any infinite computable ascending or descending chains. (The existence of such orders was proved independently by Denisov and Tennenbaum and a proof can be found in [2].) The fact that $L$ has no infinite descending chains means that it satisfies wqo in REC. However, the fact that $L$ has no infinite computable ascending chains means that $L$ does not satisfy wqo(set) in REC.

Our use of an order which is not really a wqo but only looks like a wqo in REC is necessary in the proof of Lemma 3.7. To see why this is so, consider the following result from Herrmann [4]. We have stated the result in a slightly different form than in [4], but an examination of Herrmann's proof shows that it also yields this statement. 
Theorem 3.8 (Herrmann). Every computable partial order has either a computable infinite ascending sequence or a $\Delta_{2}^{0}$ infinite descending sequence or a $\Pi_{2}^{0}$ infinite antichain.

Let $\left(P, \leq_{P}\right)$ be a computable partial order which is a real wqo so that $P$ has neither an infinite antichain nor an infinite descending sequence. We claim that $P$ satisfies wqo(set) in REC. Fix any map $f: \mathbb{N} \rightarrow P$. Since REC $=\mathrm{RT}_{<\infty}^{1}$, we can assume as in the proof of Lemma 3.3 that the range of $f$ is infinite and that $f$ is one-to-one. We define an auxiliary partial order $\leq_{f}$ with domain $\mathbb{N}$ such that $n<_{f} m$ if and only if $f(n)<_{P} f(m)$. By Herrmann's result, $\leq_{f}$ has a computable infinite increasing sequence $n_{0}<_{f} n_{1}<_{f} \cdots$. As in the proof of Lemma 3.3, this infinite increasing sequence yields an infinite set $A$ which satisfies wqo(set) for the map $f$.

Theorem 3.9. REC $\forall \neq w q o($ anti $) \rightarrow$ wqo and hence $\mathrm{RCA}_{0} \forall w q o($ anti $) \rightarrow$ wqo.

Proof. We present the proof of this theorem in considerable detail and trust the reader to fill in similar details on subsequent proofs. We build a computable partial order $\left(P, \leq_{P}\right)$ in stages. At stage $s$, we specify a finite set $P_{s}$ and a partial order on $P_{s}$. Once the partial order on $P_{s}$ is defined at stage $s$, we cannot change it later in the construction. At the end of the construction, the domain of $P$ (which is $\bigcup P_{s}$ ) will be equal to $\mathbb{N}$, and hence $\left(P, \leq_{P}\right)$ will be a computable partial order. As previously mentioned, we let $n \perp m$ denote that $n$ and $m$ are incomparable in $P$. $P$ will have the stronger property that $n \perp m$ if and only if $n$ and $m$ are incompatible: $\neg \exists z\left(z \leq_{P} n \wedge z \leq_{P} m\right)$.

To make sure that $\left(P, \leq_{P}\right)$ has no computable infinite descending chains and no computable infinite antichains, we meet the following requirements.

$$
\begin{gathered}
\mathcal{R}_{e}: \varphi_{e} \text { total } \rightarrow \exists n<_{\mathbb{N}} m\left(\varphi_{e}(n) \leq_{P} \varphi_{e}(m) \vee \varphi_{e}(n) \perp \varphi_{e}(m)\right) \\
\mathcal{S}_{e}: \varphi_{e} \text { total } \rightarrow \exists n \neq m\left(\varphi_{e}(n) \leq_{P} \varphi_{e}(m)\right) .
\end{gathered}
$$

The priority order on these requirements is $\mathcal{R}_{0}, \mathcal{S}_{0}, \mathcal{R}_{1}, \mathcal{S}_{1}, \ldots$ To make sure that $\left(P, \leq_{P}\right)$ is not a wqo in REC, we need to construct a computable function $f$ such that for all $i<j$, either $f(i) \perp f(j)$ or $f(j)<_{P} f(i)$. Our function $f$ is the simplest possible: $f(n)=n$. That is, if we just list the element of $P$ in $\leq_{\mathbb{N}}$ increasing order, we get a computable witness that $\left(P, \leq_{P}\right)$ is not a wqo.

The basic module for $\mathcal{R}_{e}$ is as follows.

1. Put down two incomparable points $q_{0}^{e}$ and $q_{1}^{e}$. Force all lower priority requirements to place new points in $P$ below $q_{0}^{e}$.

2. Wait for stage $s$ and a number $x$ such that $\varphi_{e, s}(x)=y$ for some $y$ with $y \leq_{P} q_{0}^{e}$.

3. For all stages $t>s$, force lower priority requirements to place new points in $P$ below $q_{1}^{e}$ instead of below $q_{0}^{e}$. That is, $\mathcal{R}_{e}$ forbids putting any points below $q_{0}^{e}$ after the current stage.

This module will succeed, at least in isolation from the other strategies. If there is never convergence as in 2 , then $\varphi_{e}$ enumerates only points not below $q_{0}^{e}$. Since there are only 
finitely many such points, $\varphi_{e}$ cannot be an infinite descending chain. If there is convergence as in 2 , then $\varphi_{e}$ enumerates at least one point below $q_{0}^{e}$. By 3 , the number of points below $q_{0}^{e}$ is finite, so $\varphi_{e}$ cannot be an infinite descending chain.

The basic module for $\mathcal{S}_{e}$ is as follows.

1. Put down a single point $q^{e}$. Force all lower priority requirements to place new points in $P$ below $q^{e}$.

2. Wait for stage $s$ and a number $x$ such that $\varphi_{e, s}(x)=y$ for some $y$ with $y \leq_{P} q^{e}$.

3. Pick a point $z \leq_{P} y$ which is currently minimal in $P$. For all stages $t>s$, force lower priority requirements to place new points in $P$ below $z$. That is, $\mathcal{S}_{e}$ requires lower priority requirements to work in the cone below $z$ after the current stage. (The only reason for choosing the point $z$ is to make the order relation easier to describe in the general construction.)

Again, we explain why this module succeeds in isolation from the other strategies. If there is no convergence as in 2 , then $\varphi_{e}$ enumerates only points which are not below $q^{e}$. Since there are only finitely many such points, $\varphi_{e}$ cannot enumerate an infinite antichain. If there is convergence as in 2 , then $\varphi_{e}$ enumerates at least one point $y$ above $z$. Any element incomparable with this point $y$ cannot be below $z$. In this case, by 3 there are only finitely many points not below $z$, so $\varphi_{e}$ cannot enumerate an infinite antichain.

There is an obvious source of conflict between these strategies, but it can easily be resolved with a finite injury construction. We present the formal construction using a tree of strategies, although such a tree is not necessary. However, it will be useful for one of the corollaries below to have the notation given by this tree. The tree of strategies $T$ is the full binary branching tree on the symbols $w$ and $d$ with the $d$ outcome to the left of the $w$ outcome. More formally, we define $T$ inductively. The empty string $\lambda$ is in $T$ and is an $\mathcal{R}_{0}$ node. If $\alpha$ is an $\mathcal{R}_{e}$ node in $T$, then $\alpha * d$ and $\alpha * w$ are both $\mathcal{S}_{e}$ nodes in $T$ with $\alpha * d$ to the left of $\alpha * w$. If $\alpha$ is an $\mathcal{S}_{e}$ node in $T$, then both $\alpha * d$ and $\alpha * w$ are $\mathcal{R}_{e+1}$ nodes in $T$ with $\alpha * d$ to the left of $\alpha * w$. For $\alpha \neq \beta \in T$, we say that $\alpha$ has higher priority than $\beta$ if $\alpha \sqsubset \beta$ or $\alpha(i)=d$ and $\beta(i)=w$ where $i$ is the least value for which $\alpha(i) \neq \beta(i)$. If $\alpha \in T$ and $\alpha \neq \lambda$, then $\alpha^{-}$denotes the node in $T$ formed by removing the last element of $\alpha$.

We can now present the formal construction. At stage 0 , the current true path $\delta_{0}$ in $T$ consists only of the node $\lambda$. The $\lambda$ strategy defines $q_{0}^{\lambda}=0$ and $q_{1}^{\lambda}=1$, adds these two points to $P$ and makes them incomparable in $\leq_{P}$. Also, $\lambda$ defines $u_{\lambda}=q_{0}^{\lambda}$. The parameter $u_{\lambda}$ is used to tell lower priority requirements which cones they have to place their points in. We end stage 0 and say that the strategy $\lambda$ was eligible to act at stage 0 .

At stage $t>0$, we define the current true path $\delta_{t}$ in $T$ such that $\left|\delta_{t}\right|=t$. We define $\delta_{t}$ by induction using a sequence of approximations $\delta_{t, s}$, for $s \leq t$, such that $\left|\delta_{t, s}\right|=s$ and $\delta_{t, s} \sqsubset \delta_{t, s+1}$. We set $\delta_{t}=\delta_{t, t}$. We begin with $\delta_{t, 0}=\lambda$. To proceed by induction, assume that $\delta_{t, s}=\alpha$. We describe the action taken by $\alpha$ and how to define $\delta_{t, s+1}($ if $s<t)$.

If $\alpha$ is an $\mathcal{R}_{e}$ strategy, then proceed as follows. The parameter $u_{\alpha^{-}}$denotes the element of $P$ below which $\alpha$ must place its new points, if this is the first stage at which $\alpha$ is eligible to act. Notice that step 1 will never apply to $\lambda$ since $\lambda$ was eligible to act at stage 0 . Therefore, we do not need to worry that $\lambda^{-}$is undefined. 
1. If $\alpha$ has been on the current true path before, then skip to step 2. Otherwise, let $q_{0}^{\alpha}$ and $q_{1}^{\alpha}$ be the $\leq_{\mathbb{N}^{-}}$least numbers not in $P$ yet. Make them incomparable with each other in $\leq_{P}$, below $u_{\alpha^{-}}$, and incomparable with all points which are not $\geq_{P} u_{\alpha^{-}}$. Set $u_{\alpha}=q_{0}^{\alpha}$. If $s=t$, then end the stage, and otherwise, go to step 2 .

2. Check if there is an $x \leq t$ such that $\varphi_{e, t}(x)$ converges to a node $\leq_{P} q_{0}^{\alpha}$. If not, then leave $u_{\alpha}=q_{0}^{\alpha}$ and let $\delta_{t, s+1}=\alpha * w$. If there is such an $x$, then redefine $u_{\alpha}=q_{1}^{\alpha}$ and let $\delta_{t, s+1}=\alpha * d$. (Of course, once we have seen this convergence and redefined $u_{\alpha}$, then we do not redefine it again at future stages.)

If $\alpha$ is an $\mathcal{S}_{e}$ node, then proceed as follows.

1. If $\alpha$ has been on the current true path before, then skip to step 2. Otherwise, let $q^{\alpha}$ be the $\leq_{\mathbb{N}^{-}}$least number not in $P$ yet. Place $q^{\alpha}$ below $u_{\alpha^{-}}$and incomparable with all points which are not $\geq_{P} u_{\alpha^{-}}$. Let $u_{\alpha}=q^{\alpha}$. If $s=t$, then end the stage, and otherwise, go to step 2.

2. Check if there is an $x \leq t$ such that $\varphi_{e, t}(x)$ converges to an element $y \leq_{P} q^{\alpha}$. If not, then leave $u_{\alpha}=q^{\alpha}$ and let $\delta_{t, s+1}=\alpha * w$.

3. If there is such an $x$ and $\alpha * d$ was on the current true path at stage $t-1$ (that is, $\left.\alpha * d \sqsubseteq \delta_{t-1}\right)$, then let $u_{\alpha}$ be the same as it was at stage $t-1$ and let $\delta_{t, s+1}=\alpha * d$. (This case corresponds to the situation in which we have already diagonalized and just want to continue the action described at stage $t-1$.)

4. If there is such an $x$ and $\alpha * d$ was not on the current true path at stage $t-1$ (that is, $\left.\alpha * d \nsubseteq \delta_{t-1}\right)$, then consider the least such $x$ and let $\varphi_{e}(x)=y$. Let $z$ be any point such that $z \leq_{P} y$ and there are currently no points in $P$ below $z$. Redefine $u_{\alpha}=z$ and let $\delta_{t, s+1}=\alpha * d$.

This completes the description of the construction of $P$. We sketch the verification that the construction succeeds. First, we claim that if $\alpha$ is first eligible to act at stage $t$, then at stage $t$, there are no points below $u_{\alpha^{-}}$. This is proved by induction on $t$ and on the nodes $\alpha$ on $\delta_{t}$. If $\alpha$ is on the current true path for the first time at stage $t$, then it is for one of three reasons: $\alpha^{-}$is first on the current true path at stage $t, \alpha=\alpha^{-} * d$ and $\alpha^{-}$has always taken the $\alpha^{-} * w$ outcome before, or $|\alpha|=t$. It is straightforward to verify in each case that $u_{\alpha^{-}}$ has no nodes below it before $\alpha$ acts.

Second, the current true path can only move left between consecutive stages of the construction. Therefore, the initialization of lower priority strategies is automatically taken care of by moving to strategies which have never acted before. Furthermore, this property means that we can define the true path as the pointwise limit of the current true paths visited during the construction. If $\alpha$ is on the true path, then there is a stage $s$ such that $\alpha \sqsubseteq \delta_{t}$ (that is, $\alpha$ is on the current true path) for every stage $t \geq s$. The module for a strategy $\alpha$ on the true path succeeds exactly as in the informal description of the modules working in isolation.

Third, notice that during stage $t$ of the construction, we never place a new point above a point which is already in $P$. Since we place points in $P$ in $\leq_{\mathbb{N}}$ increasing order, this means that the function $f(n)=n$ is a computable witness that $P$ is not a wqo. 
Corollary 3.10. REC $\not \models w q o($ anti $) \rightarrow w q o($ ext $)$ and $\mathrm{RCA}_{0} \nvdash w q o($ anti $) \rightarrow w q o(e x t)$.

Proof. Let $\leq_{P}$ be the computable partial order from the proof of Theorem 3.9. We prove that there is a computable linear extension $\leq_{L}$ of $\leq_{P}$ and an infinite descending chain in $\leq_{L}$ given by a computable function $f$. Before describing the linear extension, we note three properties of the construction of $P$. First, if $x<_{P} q_{0}^{\alpha}$ for some $\mathcal{R}_{e}$ strategy $\alpha$, then $x$ is placed in the partial order by a strategy $\beta$ such that $\alpha * w \sqsubseteq \beta$. Second, if $x<_{P} q_{1}^{\alpha}$ for some $\mathcal{R}_{e}$ strategy $\alpha$, then $x$ is placed in the partial order by a strategy $\beta$ such that $\alpha * d \sqsubseteq \beta$. Third, if $x<_{P} q^{\alpha}$ for some $\mathcal{S}_{e}$ strategy $\alpha$, then $x$ is placed in the partial order by a strategy $\beta$ such that $\alpha \sqsubset \beta$. These properties are all easily verified by induction on the stages of the construction.

We next assign each point $x$ in $P$ to a unique node in the tree of strategies from the proof of Theorem 3.9. If $x=q_{0}^{\alpha}$ for some $\mathcal{R}_{e}$ strategy $\alpha$, then $x$ is assigned to $\alpha * w$. If $x=q_{1}^{\alpha}$ for some $\mathcal{R}_{e}$ strategy $\alpha$, then $x$ is assigned to $\alpha * d$. If $x=q^{\alpha}$ for some $\mathcal{S}_{e}$ strategy $\alpha$, then $x$ is assigned to $\alpha * w$. We let $p_{\alpha}$ denote the unique element (if any) of $P$ assigned to $\alpha$. Notice that every point in $P$ is assigned to some $\alpha$. The linear order $\leq_{L}$ is defined by $p_{\beta} \leq_{L} p_{\gamma}$ if and only if $\gamma \sqsubseteq \beta$ or $\beta$ is to the left of $\gamma$. Since $\leq_{L}$ just copies the Kleene-Brouwer order on the tree of strategies, it is clearly a linear order on $P$.

We need to see that $\leq_{L}$ is a linear extension of $\leq_{P}$. We show by induction on $t$ that $\leq_{L}$ is a linear extension of $\leq_{P}$ on the points of $P$ at the end of stage $t$. At the end of stage $0, P$ contains only the incomparable points $q_{0}^{\lambda}$ and $q_{1}^{\lambda}$. Therefore, our induction holds at stage 0 . We assume the induction holds at stage $t$ and we show it holds at stage $t+1$ by induction on the strategies in the current true stage. That is, we examine how points are added during stage $t+1$ and show that $\leq_{L}$ extends $\leq_{P}$ after each addition. Suppose a strategy $\alpha$ adds points to $P$ at stage $t+1$ and that before $\alpha$ adds its points, $\leq_{L}$ is an extension of $\leq_{P}$. We split into cases depending on what type of strategy $\alpha$ is and whether it is below a $d$ or $w$ outcome.

Consider the case when $\alpha$ is an $\mathcal{R}_{e}$ strategy and $\alpha=\alpha^{-} * w$. Then, $\alpha^{-}$is an $\mathcal{S}_{e-1}$ strategy and in $\leq_{P}$, we add $q_{0}^{\alpha}$ and $q_{1}^{\alpha}$ as incomparable points immediately below $q^{\alpha^{-}}$. Therefore, it suffices to show that $q_{0}^{\alpha}$ and $q_{1}^{\alpha}$ are $<_{L} q^{\alpha^{-}}$. In our notation of assigning elements of $P$ to strategies, we have $q^{\alpha^{-}}=p_{\alpha}, q_{0}^{\alpha}=p_{\alpha * w}$ and $q_{1}^{\alpha}=p_{\alpha * d}$. Since $\alpha \sqsubset \alpha * w$ and $\alpha \sqsubset \alpha * d$, we have that $q_{0}^{\alpha}<_{L} q^{\alpha^{-}}$and $q_{1}^{\alpha}<_{L} q^{\alpha^{-}}$as required. A similar proof works in the cases when $\alpha$ is an $\mathcal{S}_{e}$ strategy and either $\alpha=\alpha^{-} * w$ or $\alpha=\alpha^{-} * d$.

It remains to examine the case when $\alpha$ is an $\mathcal{R}_{e}$ strategy and $\alpha=\alpha^{-} * d$. In this case, $\alpha$ adds the points $q_{0}^{\alpha}$ and $q_{1}^{\alpha}$ to $P$ immediately below some point $z \leq_{P} q^{\alpha^{-}}$. Therefore, it suffices to show that $q_{0}^{\alpha}$ and $q_{1}^{\alpha}$ are $<_{L} z$. By the properties listed in the first paragraph of this proof, $z$ is added to $P$ by some strategy $\beta$ with $\alpha^{-} \sqsubseteq \beta$. Since $\alpha=\alpha^{-} * d$ has never been eligible to act before this stage, we cannot have $\alpha \sqsubseteq \beta$. Therefore, either $\beta=\alpha^{-}$or $\alpha^{-} * w \sqsubseteq \beta$. If $\beta=\alpha^{-}$, then $z=q^{\alpha^{-}}$and so $z=p_{\alpha^{-} * w}$. Since $\alpha=\alpha^{-} * d$ is to the left of $\alpha^{-} * w$, we have that $\alpha * w$ and $\alpha * d$ are to the left of $\alpha^{-} * w$. Therefore, $q_{0}^{\alpha}=p_{\alpha * w}$ and $q_{1}^{\alpha}=p_{\alpha * d}$ are $<_{L} q^{\alpha^{-}}=p_{\alpha^{-* w}}$ as required. The last remaining case is when $\alpha^{-} * w \sqsubseteq \beta$. But, then $\alpha$ is to the left of $\beta$ and a similar argument shows $q_{0}^{\alpha}$ and $q_{1}^{\alpha}$ are $<_{L} z$ as required.

We still need to define a computable function $f: \mathbb{N} \rightarrow P$ such that for all $t, f(t+1)<_{L} f(t)$. Let $t$ be any stage of the construction. There is a last strategy $\alpha_{t}$ which is eligible to act at this stage and since $\left|\alpha_{t}\right|=t, t$ is the first stage at which $\alpha_{t}$ is eligible to act. (Technically, 
$\alpha_{t}$ is equal to $\delta_{t}$, but we use the notation $\alpha_{t}$ to distinguish the strategy from the current true path.) Therefore, $\alpha_{t}$ adds at least one point to $P$ and defines $u_{\alpha_{t}}$ to be one of the new points it has added to $P$. Furthermore, in the notation introduced above, $u_{\alpha_{t}}$ is always equal to $p_{\alpha_{t} * w}$. We define $f(t)=u_{\alpha_{t}}=p_{\alpha_{t} * w}$.

It is clear that $f$ is computable. To verify that $f(t+1)<_{L} f(t)$, we split into two cases. First, if $\alpha_{t+1}^{-} \neq \alpha_{t}$, then $\alpha_{t+1}$ is to the left of $\alpha_{t}$ in the tree of strategies. (This follows since the current true path in the construction only moves to the left.) Therefore, $\alpha_{t+1} * w$ is to the left of $\alpha_{t} * w$ and so $f(t+1)=p_{\alpha_{t+1} * w}<_{L} p_{\alpha_{t} * w}=f(t)$. Second, assume that $\alpha_{t+1}^{-}=\alpha_{t}$. If $\alpha_{t+1}=\alpha_{t} * w$, then $\alpha_{t} * w \sqsubset \alpha_{t+1} * w$ and so $f(t+1)=p_{\alpha_{t+1} * w}<_{L} p_{\alpha_{t} * w}=f(t)$. Otherwise, we have $\alpha_{t+1}=\alpha_{t} * d$. In this case, we first assume that $\alpha_{t}$ is an $\mathcal{S}_{e}$ strategy. We know $f(t)$ is set to the value of $u_{\alpha_{t}}$ at the end of stage $t$. Thus, $f(t)=q^{\alpha_{t}}$. However, $\alpha_{t}$ was the last strategy to act at stage $t$ and so no strategy has placed any points below $q^{\alpha_{t}}$. Therefore, when $\alpha_{t}$ goes to the $\alpha_{t} * d$ outcome at stage $t+1$, the only choice for $u_{\alpha_{t}}$ at stage $t+1$ is again $q^{\alpha_{t}}$. Therefore, $\alpha_{t+1}$ defines $f(t+1)=u_{\alpha_{t+1}}$ to be a point $<_{P} q^{\alpha_{t}}=f(t)$. Since $\leq_{L}$ extends $\leq_{P}$, we have $f(t+1)<_{L} f(t)$. The last remaining case is when $\alpha_{t+1}=\alpha_{t} * d$ and $\alpha_{t}$ is an $\mathcal{R}_{e}$ strategy. Then $f(t)=q_{0}^{\alpha_{t}}$ and $\alpha_{t+1}$ places its new points $<_{P} q_{1}^{\alpha_{t}}$. Again, because $\leq_{L}$ extends $\leq_{P}$, we have $f(t+1)<_{L} q_{1}^{\alpha_{t}}$. However, we set $q_{1}^{\alpha_{t}}<_{L} q_{0}^{\alpha_{t}}$ since $q_{1}^{\alpha}=p_{\alpha * d}, q_{0}^{\alpha}=p_{\alpha * w}$ and $\alpha * d$ is to the left of $\alpha * w$. Therefore, $f(t+1)<_{L} f(t)$ as required. This completes the proof that $f$ gives a computable infinite descending chain in $\leq_{L}$.

The proof of Theorem 3.9 can be strengthened to show that $\mathrm{WKL}_{0}$ does not suffice to prove wqo(anti) $\rightarrow$ wqo. The technique used to prove this stronger result is similar to the methods used in [3] and the reader is referred these for more applications of this method.

Theorem 3.11. $\mathrm{WKL}_{0} \nvdash w q o($ anti $) \rightarrow$ wqo.

Proof. To prove this theorem, we show that a particular model (or rather a particular type of model) of $\mathrm{WKL}_{0}$ cannot be a model of the implication wqo(anti) $\rightarrow$ wqo. Formally, we show that if $X_{0}, X_{1}, \ldots$ is a sequence of uniformly $\Delta_{2}^{0}$, uniformly low sets, then we can construct a computable partial order $\leq_{P}$ which meets the requirements:

$$
\begin{gathered}
\mathcal{R}_{\langle e, i\rangle}: \varphi_{e}^{X_{i}} \text { total } \rightarrow \exists n<_{\mathbb{N}} m\left(\varphi_{e}^{X_{i}}(n) \leq_{P} \varphi_{e}^{X_{i}}(m) \vee \varphi_{e}^{X_{i}}(n) \perp \varphi_{e}^{X_{i}}(m)\right) \\
\mathcal{S}_{\langle e, i\rangle}: \varphi_{e}^{X_{i}} \text { total } \rightarrow \exists n \neq m\left(\varphi_{e}^{X_{i}}(n) \leq_{P} \varphi_{e}^{X_{i}}(m)\right)
\end{gathered}
$$

The motivation for meeting these requirements is that there is such a sequence of sets $X_{i}$ for which the $\omega$-model $\mathcal{A}$ with second order part $\left\{Y \mid \exists i\left(Y \leq_{T} X_{i}\right)\right\}$ is a model of $\mathrm{WKL}_{0}$. Since $\leq_{P}$ is a computable partial order, it is an element of $\mathcal{A}$. Furthermore, the $\mathcal{R}_{\langle e, i\rangle}$ requirements show that $\leq_{P}$ has no infinite descending sequences in $\mathcal{A}$ and the $\mathcal{S}_{\langle e, i\rangle}$ requirements show that $\leq_{P}$ has no infinite antichains in $\mathcal{A}$. Therefore, $\leq_{P}$ satisfies wqo(anti) in $\mathcal{A}$. To make sure that $\leq_{P}$ does not satisfy wqo in $\mathcal{A}$, we construct a computable function $f$ such that for all $i<j$, either $f(i) \perp f(j)$ or $f(j)<_{P} f(i)$, so we even have a computable witness that $\leq_{P}$ does not satisfy wqo. As above, our function is $f(n)=n$.

This argument is almost identical to the argument in Theorem 3.9 except that we may finitely often switch between the outcomes $w$ and $d$ rather than just moving from $w$ to $d$ and never returning to $w$. The argument is still finite injury, but it may take more than one 
(but still finitely many) attempts to successfully diagonalize. Although this argument can be given using a tree of strategies as in Theorem 3.9, it is probably easiest to formalize in a more straightforward manner. The main point of using the tree of strategies in the proof of Theorem 3.9 was to make the proof of Corollary 3.10 easier. We present the basic strategies for the $\mathcal{R}_{\langle e, i\rangle}$ and $\mathcal{S}_{\langle e, i\rangle}$ requirements and after a brief discussion of their interaction, leave the formal details to the reader. The requirements can be given any priority ordering of order type $\omega$, for example giving $\mathcal{R}_{\langle e, i\rangle}$ the $2\langle e, i\rangle$ priority and giving $\mathcal{S}_{\langle e, i\rangle}$ the $2\langle e, i\rangle+1$ priority.

We begin our discussion with the action taken for the $\mathcal{S}_{\langle e, i\rangle}$ requirement. As before, when $\mathcal{S}_{\langle e, i\rangle}$ is first eligible to act (say at stage $s$ ), it picks a new point $q^{\langle e, i\rangle}$ and places it in the partial order below a point dictated by the higher priority requirements. Let $n$ denote the number of points in $P$ at stage $s . \mathcal{S}_{\langle e, i\rangle}$ forces all lower priority requirements to work below $q^{\langle e, i\rangle}$ until a stage $t>s$ such that all of the computations $\varphi_{e}^{X_{i}}(0), \ldots, \varphi_{e}^{X_{i}}(n)$ appear to converge at stage $t$. (Notice that we are now approximating the $\Delta_{2}^{0}$ set $X_{i}$, so we are really looking at computations of the form $\varphi_{e, t}^{X_{i, t}}(x)$. For simplicity of notation, we drop explicit mention of the stage from these computations.) At stage $t$, there are two possibilities: either these computations all converge to points which are not below $q^{\langle e, i\rangle}$, in which case at least two of these computations must converge to the same value and so $\mathcal{S}_{\langle e, i\rangle}$ is currently satisfied, or there is a least $x \leq_{\mathbb{N}} n$ such that $\varphi_{e}^{X_{i}}(x)$ appears to converge to a point $y \leq_{P} q^{\langle e, i\rangle}$. In the former case, $\mathcal{S}_{\langle e, i\rangle}$ takes no action and continues to force lower priority requirements to build below $q^{\langle e, i\rangle}$. In the latter case, $\mathcal{S}_{\langle e, i\rangle}$ picks a currently $\leq_{P}$ minimal point $z$ below $y$ and requires all lower priority requirements to work below $z$ at stages $\geq t$.

The main difference between this construction and the one in the proof of Theorem 3.9 is that our approximation of $X_{i}$ may change on small values and hence our apparent computations for $\varphi_{e}^{X_{i}}(0), \ldots, \varphi_{e}^{X_{i}}(n)$ may diverge again at a stage $\geq t$. If these computations change, then $\mathcal{S}_{\langle e, i\rangle}$ initializes all requirements of lower priority and starts over again with the same witness $q^{\langle e, i\rangle}$. That is, it waits for the computations $\varphi_{e}^{X_{i}}(0), \ldots, \varphi_{e}^{X_{i}}(n)$ to converge to new values and either takes no action (if it appears that $\mathcal{S}_{\langle e, i\rangle}$ is satisfied) or it picks a new value of $z$ and forces lower priority requirements to work below this new value of $z$. The obvious worry about this strategy is that it might repeat itself infinitely many times. However, this is the point at which we can appeal to the fact that $X_{i}$ is low. Because $X_{i}$ is low, $0^{\prime}$ knows whether $\varphi_{e}^{X_{i}}(0), \ldots, \varphi_{e}^{X_{i}}(n)$ really converge or not. Therefore, we have a $\Delta_{2}^{0}$ approximation to the question of whether they converge. We alter the above argument by making $\mathcal{S}_{\langle e, i\rangle}$ wait for a stage $t>s$ such that the computations $\varphi_{e}^{X_{i}}(0), \ldots, \varphi_{e}^{X_{i}}(n)$ converge and that our $\Delta_{2}^{0}$ approximation to whether these computations really converge says that they do in fact converge. Now we are in the situation that either these computations really do converge, in which case at some finite stage we see their real values and these values never change, or they do not all converge, in which case there is a finite stage after which we never believe that they all converge and so $\mathcal{S}_{\langle e, i\rangle}$ takes no action after that stage. This description makes it clear that the action of $\mathcal{S}_{\langle e, i\rangle}$ is finitary, and hence (assuming that $\mathcal{S}_{\langle e, i\rangle}$ is only initialized finitely often) it only initialized the lower priority requirements finitely often.

The basic strategy for the $\mathcal{R}_{\langle e, i\rangle}$ requirement is similarly modified from the proof of Theorem 3.9. When $\mathcal{R}_{\langle e, i\rangle}$ is first eligible to act (or at the first stage at which it is eligible to act after being initialized), it picks two new points $q_{0}^{\langle e, i\rangle}$ and $q_{1}^{\langle e, i\rangle}$ and forces lower priority 
requirements to work below $q_{0}^{\langle e, i\rangle}$. Let $n$ denote the number of points in $P$ at stage $s . \mathcal{R}_{\langle e, i\rangle}$ waits for a stage $t>s$ at which all the computations $\varphi_{e}^{X_{i}}(0), \ldots, \varphi_{e}^{X_{i}}(n)$ appear to converge. There are two cases: either none of the these computations converge to a point below either $q_{0}^{\langle e, i\rangle}$ or $q_{1}^{\langle e, i\rangle}$ (in which case at least two of the computations must converge to the same point so $\mathcal{R}_{\langle e, i\rangle}$ currently is satisfied) or there is a least $x \leq_{\mathbb{N}} n$ for which $\varphi_{e}^{X}(x)$ appears to converge to a point below one of $q_{0}^{\langle e, i\rangle}$ or $q_{1}^{\langle e, i\rangle}$. In the former case, $\mathcal{R}_{\langle e, i\rangle}$ takes no new action. In the latter case, assume that $\varphi_{e}^{X_{i}}(x)=y$ and $y \leq_{P} q_{j}^{\langle e, i\rangle}$. $\mathcal{R}_{\langle e, i\rangle}$ now forces all lower priority requirements to work below $q_{1-j}^{\langle e, i\rangle}$.

Just as with the $\mathcal{S}_{\langle e, i\rangle}$ strategy, the apparent computations $\varphi_{e}^{X_{i}}(0), \ldots, \varphi_{e}^{X_{i}}(n)$ may later diverge or converge to different values. However, the lowness of $X_{i}$ guarantees that there is a finite stage at which either these computations settle down or there is a finite stage after which we never again believe that these computations really converge. Therefore, $\mathcal{R}_{e}^{i}$ may finitely often change its mind about whether lower priority requirements have to build below $q_{0}^{\langle e, i\rangle}$ or $q_{1}^{\langle e, i\rangle}$, but eventually, if $\varphi_{e}^{X_{i}}$ is total, then $\mathcal{R}_{e}^{i}$ eventually finds true computations and either has to perform no action to diagonalize (because $\varphi_{e}^{X_{i}}$ is not one-to-one) or sees $\varphi_{e}^{X_{i}}(x)=y \leq_{P} q_{j}^{\langle e, i\rangle}$ for a $\leq_{\mathbb{N}}$ least value of $x$ and forces all lower priority requirements to build below $q_{1-j}^{\langle e, i\rangle}$ forever.

As in the proof of Theorem 3.9, there is an obvious source of conflict between these requirements. However, here we have described the solution to this conflict in terms of initializing lower priority strategies. Since the action of each $\mathcal{S}_{e}^{i}$ and $\mathcal{R}_{e}^{i}$ strategy is finitary, each requirement eventually reached a stage after which it is never initialized and henceforth it behaves exactly as in the description above. This completes the description of the construction and the proof of Theorem 3.11.

We next examine how wqo and wqo(ext) are related. $\mathrm{RCA}_{0}$ suffices to prove the implications $\mathrm{wqo} \rightarrow \mathrm{wqo}($ ext $)$ and wqo(set) $\rightarrow$ wqo(ext).

Lemma 3.12. $\mathrm{RCA}_{0} \vdash w q o \rightarrow w q o(e x t)$.

Proof. Fix a wqo $(Q, \preceq)$ and assume that $Q$ has a linear extension $\left(L, \leq_{L}\right)$ which is not well founded. Recall that this means $L$ is a linear extension of $Q / \sim$ (where $x \sim y$ if and only if $x \preceq y$ and $y \preceq x)$. Fix a function $f: \mathbb{N} \rightarrow L$ such that for all $i<j, f(j)<_{L} f(i)$. Because $\leq_{L}$ is an extension of $\preceq, f(j)<_{L} f(i)$ implies that either $f(j) \prec f(i)$ or $f(j) \perp f(i)$. Therefore, viewing $f$ as a map from $\mathbb{N}$ into $Q$, we see that $f$ contradicts the fact that $Q$ is a wqo.

Corollary 3.13. $\mathrm{RCA}_{0} \vdash w q o($ set $) \rightarrow w q o($ ext $)$.

Proof. This corollary follows from Lemma 3.12 together with the fact that $\mathrm{RCA}_{0} \vdash$ wqo $($ set $) \rightarrow$ wqo.

We also want to examine the implication wqo $($ ext $) \rightarrow$ wqo. One method for trying to prove this implication for partial orders is as follows. (The general case for quasi-orders follows from this special case.) Let $\leq_{P}$ be a partial order with domain $\mathbb{N}$ which does not satisfy wqo and let $f: \mathbb{N} \rightarrow P$ be a function which witnesses that $P$ does not satisfy wqo. That is, if $n<_{\mathbb{N}} m$, then $f(n) \not_{P} f(m)$. We would like to define a linear extension of the partial order $<_{P}$ under 
which $f$ enumerates an infinite descending chain. Such a linear extension would show that $P$ does not satisfy wqo(ext) and hence would finish our proof that wqo(ext) $\rightarrow$ wqo. We begin to construct such a linear extension by defining a binary relation $R$ on $P$ : $x R y$ holds if and only if either $x<_{P} y$ or there are numbers $n<_{\mathbb{N}} m$ such that $x=f(m)$ and $y=f(n)$. Let $<_{P}^{\prime}$ be the transitive closure of the relation $R$. Because $R$ is an acyclic relation (defined below), $<_{P}^{\prime}$ is a partial order extending $<_{P}$. Because we made $f(n+1) R f(n)$ hold for all $n, f$ enumerates an infinite descending chain in $<_{P}^{\prime}$. Let $<_{L}$ be any linear extension of $<_{P}^{\prime}$. Then $<_{L}$ is a linear extension of $<_{P}$ which is not well founded.

This proof sketch has three parts: defining $R$, taking the transitive closure, and extending a partial order to a linear order. The definition of $R$ can easily be done in $\mathrm{RCA}_{0}$ and $\mathrm{RCA}$ is strong enough to prove that every partial order has a linear extension (see for example [2]). However, as we show below, taking the transitive closure of an arbitrary relation requires $A C A_{0}$. Before presenting the formal result, we introduce some definitions.

Definition 3.14. $\left(\mathrm{RCA}_{0}\right)$ Let $A$ be a set and let $R \subseteq A \times A$ be a relation on $A$. A chain in $R$ is a finite sequence $\left\langle x_{0}, \ldots, x_{k}\right\rangle$ of elements of $A$ such that $x_{0} R x_{1} R \ldots R x_{k} . R$ is called acyclic if there is no chain $\left\langle x_{0}, \ldots, x_{k}\right\rangle$ in $R$ such that $x_{k} R x_{0}$. The transitive closure of $R$ is the relation $R^{\prime}$ defined by $i R^{\prime} j$ if and only if there is a chain $\left\langle x_{0}, \ldots, x_{k}\right\rangle$ in $R$ such that $x_{0}=i$ and $x_{k}=j$.

Lemma 3.15. $\left(\mathrm{RCA}_{0}\right)$ The following are equivalent:

\section{1. $\mathrm{ACA}_{0}$}

2. the transitive closure of every acyclic relation exists.

Proof. The implication from 1 to 2 follows because the transitive closure is arithmetically definable. To prove the implication from 2 to 1 , it suffices to show that the range of every one-to-one function exists. We reason within $\mathrm{RCA}_{0}$. Fix a one-to-one function $f$ and consider the set $\{b\} \cup\left\{x_{n} \mid n \in \mathbb{N}\right\} \cup\left\{y_{n} \mid n \in \mathbb{N}\right\}$. Define an acyclic relation $R$ on this set by $R=$ $\left\{\left\langle b, x_{n}\right\rangle \mid n \in \mathbb{N}\right\} \cup\left\{\left\langle x_{n}, y_{f(n)}\right\rangle \mid n \in \mathbb{N}\right\}$. By 2 , we let $R^{\prime}$ be the transitive closure of $R$. Then, $m \in \operatorname{range}(f)$ if and only if $\left\langle b, y_{m}\right\rangle \in R^{\prime}$.

Because of Lemma 3.15, we cannot naively form the transitive closure in the proof sketched above. However, notice that we do not necessarily need the transitive closure of $R$, but rather we only need some extension of $R$ which is a partial order. The ability to extend any acyclic relation to a partial order turns out to be equivalent to $\mathrm{WKL}_{0}$.

Lemma 3.16. $\left(\mathrm{RCA}_{0}\right)$ The following are equivalent:

\section{1. $\mathrm{WKL}_{0}$;}

2. every acyclic relation can be extended to a partial order.

Proof. First, we show that $\mathrm{WKL}_{0}$ suffices to prove that every acyclic relation can be extended to a partial order. Let $R$ be an acyclic relation on $A$. To simplify our notation, we assume that $A=\mathbb{N}$. We define a tree $T \subseteq 2^{<\omega}$ such that every path through $T$ codes a partial order 
extending $R$. We view all sequences $s \in 2^{<\mathbb{N}}$ as sequences of pairs of elements from $\mathbb{N}$ and we think of $s$ as giving a finite approximation $<_{s}$ to a partial order extending $R$. That is, we interpret $s(\langle n, m\rangle)=1$ as specifying $n<_{s} m$ and $s(\langle n, m\rangle)=0$ as specifying $n \nless_{s} m$. We put $s \in T$ if and only if

1. $m R n$ and $\langle m, n\rangle \in \operatorname{dom}(s)$ imply $s(\langle m, n\rangle)=1$;

2. $s(\langle l, m\rangle)=1, s(\langle m, n\rangle)=1$ and $\langle l, n\rangle \in \operatorname{dom}(s)$ imply $s(\langle l, n\rangle)=1$;

3. $\langle n, m\rangle,\langle m, n\rangle \in \operatorname{dom}(s)$ implies $s(\langle m, n\rangle)+s(\langle n, m\rangle) \leq 1$;

4. $\langle n, n\rangle \in \operatorname{dom}(s)$ implies $s(\langle n, n\rangle)=0$.

It is clear that $T$ is a tree, and we verify that it is infinite. Fix $N \in \mathbb{N}$ and for each pair $\langle n, m\rangle<N$, define $s(\langle n, m\rangle)=1$ if and only if there is a chain $\left\langle x_{0}, \ldots, x_{k}\right\rangle$ in $R$ such that each $x_{i}<N$ and $x_{0}=n$ and $x_{k}=m$. It is straightforward to verify, using the fact that $R$ is acyclic, that $s \in T$. Therefore, $T$ is infinite and by $\mathrm{WKL}_{0}$ it has a path. If $h$ is any path in $T$, then define $<_{h}$ by $n<_{h} m$ if and only if $h(\langle n, m\rangle)=1$. By our definition of $T, \leq_{h}$ is a partial order extending $R$.

Second, we show that 2 implies 1 in the statement of the lemma. To show $\mathrm{WKL}_{0}$, it suffices to consider an arbitrary pair of one-to-one functions $f, g: \mathbb{N} \rightarrow \mathbb{N}$ with disjoint ranges and show that there is a set $X$ such that for all $n, f(n) \in X$ and $g(n) \notin X$. We define our relation on the set $\{b\} \cup\left\{x_{n} \mid n \in \mathbb{N}\right\} \cup\left\{y_{n} \mid n \in \mathbb{N}\right\} \cup\left\{z_{n} \mid n \in \mathbb{N}\right\}$ by

$$
R=\left\{\left\langle x_{n}, b\right\rangle \mid n \in \mathbb{N}\right\} \cup\left\{\left\langle b, y_{n}\right\rangle \mid n \in \mathbb{N}\right\} \cup\left\{\left\langle z_{f(n)}, x_{n}\right\rangle \mid n \in \mathbb{N}\right\} \cup\left\{\left\langle y_{n}, z_{g(n)}\right\rangle \mid n \in \mathbb{N}\right\} .
$$

Because $f$ and $g$ have disjoint ranges, this relation is acyclic. By 1 , there is a partial order $<_{P}$ which extends $R$. Let $X=\left\{m \mid z_{m}<_{P} b\right\}$ and notice that $X$ is our desired separating set.

Theorem 3.17. $\mathrm{WKL}_{0} \vdash w q o(e x t) \rightarrow$ wqo.

Proof. We reason in $\mathrm{WKL}_{0}$. Fix a quasi-order $Q$ which does not satisfy wqo and let $P$ be the partial order $Q / \sim$. Since $Q$ does not satisfy wqo, neither does $P$. Therefore, we can fix a function $f: \mathbb{N} \rightarrow P$ such that for all $n<m, f(n) \not_{P} f(m)$. In particular, this means that $f$ is one-to-one. Furthermore, we can define a subsequence $g: \mathbb{N} \rightarrow P$ of $f$ as follows. Set $g(0)=f(0)$. If $g(n)=f(i)$, then let $g(n+1)=f(j)$ where $j$ is the $\leq_{\mathbb{N}}$ least number such that $i<_{\mathbb{N}} j$ and $g(n)<_{\mathbb{N}} f(j)$. Such a $j$ exists because $f$ is one-to-one. It is clear from our definition of $g$ that for all $n<m, g(n) \mathbb{L}_{P} g(m)$ and for all $n, g(n) \geq_{\mathbb{N}} n$. To show that $Q$ does not satisfy wqo(ext), it suffices to produce a linear extension $\leq_{L}$ of $\leq_{P}$ which is not well ordered. We use the function $g$ to give an infinite descending sequence in $\leq_{L}$ by making $g(n+1)<_{L} g(n)$ for all $n$.

Define an acyclic relation $R$ on $P$ by $\langle i, j\rangle \in R$ if and only if $i<_{P} j$ or there are $n \leq_{\mathbb{N}} j$ and $m \leq_{\mathbb{N}} i$ such that $g(m)=i, g(n)=j$ and $n<_{\mathbb{N}} m$. It follows from our hypothesis on $g$ that $R$ is an acyclic relation. Therefore, by Lemma 3.16, there is a partial order $<_{P}^{\prime}$ extending $R$. It is clear that $<_{P}^{\prime}$ extends $<_{P}$ and that $g$ enumerates an infinite descending sequence in $<_{P}^{\prime}$. Let $<_{L}$ be any linear extension of $<_{P}^{\prime}$ and notice that $<_{L}$ is a linear extension of $<_{P}$ such that $<_{L}$ has an infinite descending chain given by $g$. 
Corollary 3.18. $\mathrm{WKL}_{0} \vdash w q o($ ext $) \rightarrow$ wqo(anti).

Proof. This result follows from Theorem 3.17 together with the fact that $\mathrm{RCA}_{0} \vdash$ wqo $\rightarrow$ wqo(anti) (from Theorem 2.5).

Corollary 3.19. $\mathrm{WKL}_{0} \nvdash w q o($ anti $) \rightarrow w q o($ ext $)$.

Proof. By Theorem 3.17, $\mathrm{WKL}_{0} \vdash$ wqo(ext) $\rightarrow$ wqo, and by Theorem 3.11, $\mathrm{WKL}_{0} \not$ wqo(anti) $\rightarrow$ wqo.

Notice that while $\mathrm{WKL}_{0}$ suffices to prove most of the implications from wqo(ext), it does not suffice to prove wqo(ext) $\rightarrow$ wqo(set). (Recall that $W_{K L_{0}}$ is not strong enough to prove $\left.\mathrm{RT}_{<\infty}^{1} \cdot\right)$

Lemma 3.20. $\mathrm{RCA}_{0} \vdash($ wqo $($ ext $) \rightarrow$ wqo $($ set $)) \rightarrow \mathrm{RT}_{<\infty}^{1}$ and hence $\mathrm{WKL}_{0} \forall$ wqo $($ ext $) \rightarrow$ wqo(set).

Proof. Let $k$ denote the trivial partial order on $\{0, \ldots, k-1\}$ in which all elements are incomparable. $\mathrm{RCA}_{0}$ is strong enough to prove this partial order is a wqo, and hence by Lemma 3.12 this partial order satisfies wqo(ext). Consider any map $f: \mathbb{N} \rightarrow k$. Since $k$ satisfies wqo(ext), the implication wqo(ext) $\rightarrow$ wqo(set) gives an infinite set $A$ such that for all $i<j \in A, f(i) \preceq f(j)$ in the order on $k$. However, since all elements of $k$ are incomparable, $f(i) \preceq f(j)$ implies that $f(i)=f(j)$ and hence $A$ is the desired homogeneous set.

Lemma 3.20 does not rule out the possibility that $\mathrm{REC} \models$ wqo(ext $) \rightarrow$ wqo(set) since $\mathrm{REC} \models \mathrm{RT}_{<\infty}^{1}$. However, a corollary of the next theorem will eliminate this possibility.

Theorem 3.21. REC $\forall \neq w q o(e x t) \rightarrow w q o$ and hence $\mathrm{RCA}_{0} \nvdash w q o(e x t) \rightarrow w q o$.

Proof. We build a computable partial order $\left(P, \leq_{P}\right)$ with $P=\mathbb{N}$ such that every computable linear extension of $\leq_{P}$ is well ordered with respect to REC. Formally, for all $e, i \in \mathbb{N}$, we meet the requirement $\mathcal{R}_{\langle e, i\rangle}$ which says that if $\varphi_{e}$ and $\varphi_{i}$ are both total and $\varphi_{e}$ is a linear extension of $\leq_{P}$, then there exist $n<m$ such that $\varphi_{i}(n) \leq_{\varphi_{e}} \varphi_{i}(m)$. That is, if $\varphi_{e}$ is a linear extension of $\leq_{P}$, then $\varphi_{i}$ is not an infinite descending chain in the order given by $\varphi_{e}$.

Furthermore, we need to make sure that $\leq_{P}$ is not a well quasi-order with respect to REC by defining a computable function $f$ such that for all $i<j$, either $f(i) \perp f(j)$ or $f(j)<_{P} f(i)$. Our function $f$ will in fact have the stronger property that it defines a computable antichain: for all $i \neq j, f(i) \perp f(j)$.

We first describe the module for a requirement $\mathcal{R}_{\langle e, i\rangle}$ working in isolation. (In the general construction, $\mathcal{R}_{\langle e, i\rangle}$ will be working inside an interval given by higher priority requirements. If $\mathcal{R}_{\langle e, i\rangle}$ is the highest priority requirement, then this interval is defined by the least and the greatest point in our partial order. Because these points play no role in the action of $\mathcal{R}_{\langle e, i\rangle}$ other than to dictate where it takes place, we ignore them for the moment.) In order to construct our computable function $f$, we begin to define an antichain of points $f(0), f(1), \ldots$, placing one new point in this antichain at each stage of the construction. $\mathcal{R}_{\langle e, i\rangle}$ picks two new points (which are kept out of the range of $f$ ) $x_{\langle e, i\rangle}$ and $y_{\langle e, i\rangle}$ and makes them incomparable in $\leq_{P}$ with each other and with all the points in the range of $f$. Until $\varphi_{e}$ gives us an order 
on these two auxiliary points, $\mathcal{R}_{\langle e, i\rangle}$ does not believe that $\varphi_{e}$ is total and so does nothing to diagonalize. Assume that $\varphi_{e}$ eventually does give us an order on our auxiliary points and that it makes $x_{\langle e, i\rangle}<_{\varphi_{e}} y_{\langle e, i\rangle}$. After this stage, we begin to place all the new points of $P$ below $x_{\langle e, i\rangle}$. That is, we continue to build an antichain for the range of $f$, but we place these points below $x_{\langle e, i\rangle}$. (In the general construction, we also force all lower priority requirements to place their new points below $\left.x_{\langle e, i\rangle}.\right) \mathcal{R}_{\langle e, i\rangle}$ now waits for $\varphi_{i}$ to enumerate a point below $x_{\langle e, i\rangle}$. Since there are only finitely many points in $P$ which are not below $x_{\langle e, i\rangle}$, we win $\mathcal{R}_{\langle e, i\rangle}$ unless $\varphi_{i}$ picks one of these points. Once we see some $n$ for which $\varphi_{i}(n)$ converges to a point $\leq_{P} x_{\langle e, i\rangle}$, we switch our tactics again and begin to build the antichain given by $f$ above $y_{\langle e, i\rangle}$. At this point, $\mathcal{R}_{\langle e, i\rangle}$ promises to continue the tactic of allowing new points only above $y_{\langle e, i\rangle}$ for the rest of the construction. Since there are only finite many points which are not above $y_{\langle e, i\rangle}$, we have that either $\varphi_{i}$ is not total, or it has finite range, or $\varphi_{i}(m)$ eventually converges for some $m>_{\mathbb{N}} n$ with $y_{\langle e, i\rangle} \leq_{P} \varphi_{i}(m)$. If $\varphi_{e}$ really is a linear extension of $\leq_{P}$, then the numbers $n$ and $m$ are our witnesses that $\mathcal{R}_{\langle e, i\rangle}$ succeeds since $n<_{\mathbb{N}} m$ and $\varphi_{i}(n) \leq_{P} x_{\langle e, i\rangle}<_{\varphi_{e}} y_{\langle e, i\rangle} \leq_{P} \varphi_{i}(m)$, so that $\varphi_{i}(n) \leq_{\varphi_{e}} \varphi_{i}(m)$.

The general construction just iterates the above procedure. The tree of strategies is the full ternary branching tree on the letters $a, b, w$ with $a$ to the left of $b$ and $b$ to the left of $w$. The nodes $\alpha$ at level $\langle e, i\rangle$ of this tree are the strategies working for $\mathcal{R}_{\langle e, i\rangle}$. A strategy $\alpha$ keeps four parameters: two witnesses $x_{\alpha}$ and $y_{\alpha}$ which are kept out of the range of $f$, and two points $u_{\alpha}<_{P} v_{\alpha}$ which tell the requirements of lower priority to work in the interval between $u_{\alpha}$ and $v_{\alpha}$. The first time a strategy $\alpha$ is eligible to act, it picks witnesses $x_{\alpha}$ and $y_{\alpha}$, adds these point to $P$, makes them incomparable in $\leq_{P}$ and in the appropriate interval as dictated by higher priority requirements, and keeps them out of the range of $f$. While $\alpha$ is waiting for $\varphi_{e}$ to give an order to $x_{\alpha}$ and $y_{\alpha}$, it takes outcome $\alpha * w$ and sets its parameters $u_{\alpha}$ and $v_{\alpha}$ to define the same interval that $\alpha$ was given by the higher priority requirements. Once an order between these nodes is established (and without loss of generality we assume $x_{\alpha}<_{\varphi_{e}} y_{\alpha}$ ), it takes outcome $\alpha * b$ and forces future points in the range of $f$ and witnesses for lower priority requirements to be placed below $x_{\alpha}$. To pass this information to the lower priority requirements, it resets its parameter $v_{\alpha}=x_{\alpha}$ and leaves the parameter $u_{\alpha}$ unchanged. If $\varphi_{i}$ ever gives a computation $\varphi_{i}(n)$ with value $\leq_{P} x_{\alpha}$, then $\alpha$ switches to outcome $\alpha * a$ and forces future points in the range of $f$ and witnesses for lower priority requirements to be placed above $y_{\alpha}$. It passes this information to lower priority requirements by resetting its parameters so that $u_{\alpha}=y_{\alpha}$ and $v_{\alpha}$ is the upper bound passed down from the higher priority requirements. That is, $v_{\alpha}$ returns to the original value that $\alpha$ was given before it saw that $x_{\alpha}<_{\varphi_{e}} y_{\alpha}$.

The construction proceeds as follows. At stage 0 , we begin by placing 0 as the least point in $\leq_{P}$ and 1 as the greatest point in $\leq_{P}$. We define $f(0)=2$ and let $\mathcal{R}_{\lambda}$ set $x_{\lambda}=3$ and $y_{\lambda}=4$. These three points are pairwise incomparable in $\leq_{P}$ and between the greatest and least points in $\leq_{P}$. $\mathcal{R}_{\lambda}$ defines $u_{\lambda}=0$ and $v_{\lambda}=1$.

At stage $t>0$, we assume that we have already defined $f(0), \ldots, f(t-1)$ to be a finite antichain. The module for a strategy $\alpha$ that is working for $\mathcal{R}_{\langle e, i\rangle}$ and that is eligible to act is as follows. (As in the previous constructions, $\alpha^{-}$denotes the strategy of immediately higher priority than $\alpha$. Notice that for stages $t>0$, the highest priority strategy $\lambda$ will not act as in 
step 1 below, so it does not matter that $\lambda^{-}$is not defined.)

1. If this is the first stage at which $\alpha$ is eligible to act, then we let $x_{\alpha}$ and $y_{\alpha}$ be the $\leq_{\mathbb{N}^{-}}$ least numbers not in $P$ yet. $\alpha$ has been passed parameters $u_{\alpha^{-}}$and $v_{\alpha^{-}}$from $\alpha^{-}$. The points $x_{\alpha}$ and $y_{\alpha}$ are placed in $\leq_{P}$ as follows: they are incomparable with each other; if $z \leq_{P} u_{\alpha^{-}}$, then $z<_{P} x_{\alpha}, y_{\alpha}$; if $v_{\alpha^{-}} \leq_{P} z$, then $x_{\alpha}, y_{\alpha}<_{P} z$; and they are incomparable with all other points. Let $\alpha * w$ be the next strategy eligible to act. Set $u_{\alpha}=u_{\alpha^{-}}$, set $v_{\alpha}=v_{\alpha^{-}}$and pass these parameters to $\alpha * w$.

2. If $\alpha$ has been eligible to act before, then check if $\varphi_{e}$ defines an order on $x_{\alpha}$ and $y_{\alpha}$ yet. If not, then let $\alpha * w$ be the next to act and leave the parameters $u_{\alpha}$ or $v_{\alpha}$ as they are currently defined. If so, then without loss of generality, assume that $x_{\alpha}<_{\varphi_{e}} y_{\alpha}$. Set $v_{\alpha}=x_{\alpha}$ and leave $u_{\alpha}$ as it is currently defined. Proceed to step 3.

3. Check if $\varphi_{i, t}(n)$ converges for some $n \leq_{\mathbb{N}} t$ such that $\varphi_{i, t}(n) \leq_{P} x_{\alpha}$. If not, then let $\alpha * b$ be the next strategy eligible to act and pass the parameters $u_{\alpha}$ and $v_{\alpha}$ to $\alpha * b$. Otherwise, if there is such an $n$, set $u_{\alpha}=y_{\alpha}$ and set $v_{\alpha}=v_{\alpha^{-}}$. Let $\alpha * a$ the next strategy eligible to act and pass the parameters $u_{\alpha}$ and $v_{\alpha}$ to $\alpha * a$.

Once we have defined the current true path $\delta_{t}$ at this stage, we need to define $f(t)$ to be the next element of our antichain. Let $f(t)$ be the $\leq_{\mathbb{N}}$ least number not in $P$ yet and let $\alpha=\delta_{t}$ be the last strategy that was eligible to act. Place $f(t)$ in $\leq_{P}$ as follows: if $z \leq_{P} u_{\alpha}$, then $z<_{P} f(t)$; if $v_{\alpha} \leq_{P} z$, then $f(t)<_{P} z$; and $f(t)$ is incomparable with all other points in $\leq_{P}$ at this stage. This completes the description of the construction at stage $t$.

We sketch the verification that this construction succeeds. The construction is finite injury and the current true path can only move left between stages of the construction. As before, the initialization of lower priority strategies is taken care of by the movement of the current true path and we can define the true path to be the pointwise limit of the current true paths. The individual modules for requirements along the true path are satisfied just as in the informal description.

We need to see that the range of $f$ is an antichain in $P$. We prove this fact by a sequence of claims, each of which can be verified by induction on the stages of the construction. First, once a point in the range of $f$ is placed in $P$, nothing is subsequently placed above it or below it. Second, any point marked as a parameter $u_{\alpha}$ may have points from the range of $f$ above it in $\leq_{P}$, but it will never have any points from the range of $f$ below it in $\leq_{P}$. Similarly, a point marked $v_{\alpha}$ may have points from the range of $f$ below it, but it will never have any points from the range of $f$ above it. Given these claims, it is clear from the definition of $f(t)$ that when it is placed in $P$, there are no points from the range of $f$ either above it or below it. This property is then maintained for the rest of the construction.

We mention one corollary which is stated in the terminology of effective algebra rather than reverse mathematics. While similar corollaries could be stated for several of the earlier results, we mention this one in particular because it seems interesting as a statement independent from the general project of this paper. 
Corollary 3.22. There is a computable partial order $\left(P, \leq_{P}\right)$ such that $P$ has a computable infinite antichain and yet every computable linear extension of $P$ is computably well ordered.

Proof. This corollary follows directly from the proof of Theorem 3.21 since our computable function $f$ is an infinite antichain.

Corollary 3.23. REC $\not \neq w q o(e x t) \rightarrow w q o\left(\right.$ anti) and hence $\mathrm{RCA}_{0} \forall w q o($ ext $) \rightarrow$ wqo(anti).

Proof. Let $P$ be a computable partial order as in Corollary 3.22. REC is a model for the fact that $P$ satisfies wqo(ext) but is not a model for $P$ satisfying wqo(anti).

Corollary 3.24. REC $\forall \neq w q o($ ext $) \rightarrow w q o($ set $)$.

Proof. Suppose that REC $\models$ wqo(ext) $\rightarrow$ wqo(set). Since $\mathrm{RCA}_{0} \vdash$ wqo(set) $\rightarrow$ wqo, we have that $\mathrm{REC} \models$ wqo $($ ext $) \rightarrow$ wqo which contradicts Theorem 3.21.

\section{Closure properties for well quasi-orders}

If $\left(Q_{1}, \preceq_{1}\right)$ and $\left(Q_{2}, \preceq_{2}\right)$ are quasi-orders, then the product quasi-order on $Q_{1} \times Q_{2}$ is given by $\left\langle q_{1}, q_{2}\right\rangle \preceq\left\langle r_{1}, r_{2}\right\rangle$ if and only if $q_{1} \preceq_{1} r_{1}$ and $q_{2} \preceq_{2} r_{2}$. In this section, we examine how difficult it is to show that the various properties defining well quasi-orders are closed under intersection and products. That is, we consider each pair of properties $P_{1}, P_{2}$ from wqo, wqo(set), wqo(anti) and wqo(ext) and examine how difficult it is to show that if $\preceq_{1}$ and $\preceq_{2}$ are quasi-orders satisfying $P_{1}$ on $Q$, then $\preceq_{1} \cap \preceq_{2}$ satisfies $P_{2}$ on $Q$, and to show that if $\left(Q_{1}, \preceq_{1}\right)$ and $\left(Q_{2}, \preceq_{2}\right)$ are quasi-orders satisfying $P_{1}$, then the product order on $Q_{1} \times Q_{2}$ satisfies $P_{2}$. We begin by examining the closure properties under intersection and then note that by Lemma 5.20 in [7], these results hold for products as well.

Lemma 4.1. RCA $\mathrm{A}_{0}$ suffices to prove that if $\preceq_{1}$ and $\preceq_{2}$ satisfy wqo(set), then $\preceq_{1} \cap \preceq_{2}$ satisfies wqo(set).

Proof. Assume that $\preceq_{1}$ and $\preceq_{2}$ satisfy wqo(set) and fix a map $f: \mathbb{N} \rightarrow Q$. Viewing $f$ as a map from $\mathbb{N}$ into the domain of $\preceq_{1}$, there is an infinite set $A_{1}$ such that for all $i<_{\mathbb{N}} j \in A_{1}$, $f(i) \preceq_{1} f(j)$. Now consider the restriction of $f$ to $A_{1}$. There is an infinite set $A_{2} \subseteq A_{1}$ such that for all $i<_{\mathbb{N}} j \in A_{2}, f(i) \preceq_{2} f(j)$. Because $A_{2} \subseteq A_{1}$, we have that for all $i<_{\mathbb{N}} j \in A_{2}$, $f(i) \preceq_{1} f(j)$ and hence $f(i)$ is below $f(j)$ in $\preceq_{1} \cap \preceq_{2}$. (More formally, to define $A_{2}$, we enumerate $A_{1}$ as $a_{0}<_{\mathbb{N}} a_{1}<_{\mathbb{N}} \cdots$ and define a map $f^{\prime}: \mathbb{N} \rightarrow Q$ by $f^{\prime}(n)=f\left(a_{n}\right)$. We then apply wqo(set) for $\preceq_{2}$ to the map $f^{\prime}$.)

Corollary 4.2. Let $P$ be any of the properties wqo, wqo(ext), wqo(anti) or wqo(set). $\mathrm{RCA}_{0}$ is strong enough to prove that if $\preceq_{1}$ and $\preceq_{2}$ satisfy wqo(set), then $\preceq_{1} \cap \preceq_{2}$ satisfies $P$.

Proof. This corollary follows since $\mathrm{RCA}_{0}$ is strong enough to prove that wqo(set) implies each of the other properties defining well quasi-orders. 
We next show that $\mathrm{WKL}_{0}$ is not strong enough to prove the closure under intersections for any of the remaining properties. Let $X_{0}, X_{1}, \ldots$ be a sequence of uniformly $\Delta_{2}^{0}$ and uniformly low sets such that the $\omega$-model $\mathcal{A}$ with second order part $\left\{Y \mid \exists i\left(Y \leq_{T} X_{i}\right)\right\}$ is a model of $\mathrm{WKL}_{0}$. The following theorem shows that $\mathcal{A}$ is not a model for the closure of quasi-orders satisfying wqo under intersection.

Theorem 4.3. There are computable partial orders $\leq_{0}$ and $\leq_{1}$ such that both $\leq_{0}$ and $\leq_{1}$ satisfy wqo in $\mathcal{A}$ but $\leq_{0} \cap \leq_{1}$ is an infinite antichain.

Proof. We build $\leq_{0}$ and $\leq_{1}$ in stages so that each is a partial order on $\mathbb{N}$. We guarantee that each satisfies wqo in $\mathcal{A}$ by meeting the following requirements for $e, i \in \mathbb{N}$ and $j \in\{0,1\}$.

$$
\mathcal{R}_{\langle e, i, j\rangle}: \varphi_{e}^{X_{i}} \text { total and one-to-one } \rightarrow \exists k<l\left(\varphi_{e}(k) \leq_{j} \varphi_{e}(l)\right)
$$

In fact, we will have the additional property that each $\leq_{j}$ has no infinite descending chains at all (that is even outside of $\mathcal{A}$ ) since $n \leq_{j} m$ will imply $n \leq_{\mathbb{N}} m$. We make $\leq_{0} \cap \leq_{1}$ an antichain by guaranteeing that if $n<_{j} m$, then $n \nless_{1-j} m$. We refer to this requirement as our antichain requirement. We order the priority for our $\mathcal{R}_{\langle e, i, j\rangle}$ requirements in type $\omega$ by ordering the triples $\langle e, i, j\rangle$ in type $\omega$.

Consider first the basic module for the requirement $\mathcal{R}_{\langle 0,0,0\rangle} \cdot \mathcal{R}_{\langle 0,0,0\rangle}$ picks two points $q^{0}$ and $q^{1}$ and makes them incomparable in both the $\leq_{0}$ and $\leq_{1}$ orders. It forces the $\leq_{0}$ partial order to place all its points above $q^{0}$ and the $\leq_{1}$ order to place all its points above $q^{1}$. This guarantees that $q^{j} \leq_{j} x$ for all $x \neq q^{1-j}$, while $q^{j} \nless_{1-j} x$ for all $x$. We therefore are meeting our requirements for making $\leq_{0} \cap \leq_{1}$ an antichain with respect to the points $q^{0}$ and $q^{1}$. Currently, there is exactly one point (the point $q^{1}$ ) which is not $\geq_{0} q^{0}$ in the $\leq_{0}$ order. We wait for the computations $\varphi_{0}^{X_{0}}(0)$ and $\varphi_{0}^{X_{0}}(1)$ to appear to converge. If one of these computations never appears to converge, then $\mathcal{R}_{\langle 0,0,0\rangle}$ is easily won since $\varphi_{0}^{X_{0}}$ is not total. Therefore, assume that these computations do appear to converge at some stage $s$. We have two cases to consider. Either both computations appear to converge to $q^{1}$, in which case $\varphi_{0}^{X_{0}}$ does not appear to be one-to-one and so $\mathcal{R}_{\langle 0,0,0\rangle}$ takes no additional action, or one of these computations converges to a point $y$ such that $q^{0} \leq_{0} y$. At this stage, $\mathcal{R}_{\langle 0,0,0\rangle}$ initializes all lower priority requirements (that is makes them begin again with new witnesses) and forces them to work above $y$ in the $\leq_{0}$ order. Since at stage $s$ there are only finitely many points so far not above $y$ in the $\leq_{0}$ order, this guarantees that if $\varphi_{0}^{X_{0}}$ is total and one-to-one then $\mathcal{R}_{\langle 0,0,0\rangle}$ will be met.

When $\mathcal{R}_{\langle 0,0,0\rangle}$ begins to force lower priority requirements to work above $y$ in $\leq_{0}$, we also need to consider where to make lower priority requirements work in the $\leq_{1}$ order. Because each lower priority requirement works in a similar manner to $\mathcal{R}_{\langle 0,0,0\rangle}$, points are always placed in $\leq_{0}$ and $\leq_{1}$ in pairs. That is, the point $y$ is one point in a pair of points $z^{0}, z^{1}$ which were placed in the partial orders as witnesses for a lower priority requirement. Without loss of generality, we suppose that $y=z^{1}$. The points $z^{0}, z^{1}$ were made incomparable in both partial orders and so when $\mathcal{R}_{\langle 0,0,0\rangle}$ forces lower priority requirements to work above $y=z^{1}$ in the $\leq_{0}$ order, it also forces all lower priority requirements to work above the other element of the pair, in this case $z^{0}$, in the $\leq_{1}$ order. In this fashion we maintain our antichain requirements.

As in the proof of Theorem 3.11, the computations $\varphi_{0}^{X_{0}}(0)$ and $\varphi_{0}^{X_{0}}(1)$ may change at a later stage. Each time these computations change values, $\mathcal{R}_{\langle 0,0,0\rangle}$ initializes the lower priority 
requirements and picks a new value of $y \geq_{0} q^{0}$ to force lower priority requirements to work above in $\leq_{0}$ and uses the corresponding element to force requirements to work above in $\leq_{1}$. The fact that $X_{0}$ is $\Delta_{2}^{0}$ and low means that after finitely many such changes, we reach a stage at which we see the true value of the computations or after which we never believe both computations converge. Therefore, the action of $\mathcal{R}_{\langle 0,0,0\rangle}$ is finitary.

The general construction just iterates this strategy for one requirement. Because the action of a single $\mathcal{R}_{\langle e, i, j\rangle}$ strategy is finitary, the argument is finite injury. It can be formalized with a tree of strategies as in Theorem 3.9, although it is easier to formalize without the extra machinery of the tree of strategies. We describe a formal module for $\mathcal{R}_{\langle e, i, j\rangle}$ below and leave the details of combining these strategies in a finite injury argument to the reader.

Let $\alpha$ be a triple $\langle e, i, j\rangle$. The requirement $\mathcal{R}_{\alpha}$ keeps five pieces of information: two points $q_{\alpha}^{0}$ and $q_{\alpha}^{1}$ which it picks and adds to the partial orders when it is first eligible to act (and repicks when it is initialized), two parameters $u_{\alpha}^{0}$ and $u_{\alpha}^{1}$ which it uses to tell requirements of lower priority where to place their points, and a number $n_{\alpha}$ which gives the length of convergence of $\varphi_{e}^{X_{i}}$ that $\mathcal{R}_{\alpha}$ is waiting for. It requires that lower priority requirements place points in the $\leq_{k}$ order in the cone about $u_{\alpha}^{k}$. The module for $\mathcal{R}_{\alpha}$ is as follows. (We let $\mathcal{R}_{\alpha^{-}}$ denote the requirement of immediately higher priority than $\mathcal{R}_{\alpha}$.)

1. The first time $\alpha$ is eligible to act or the first time it is eligible to act after having been initialized, it takes the two $\leq_{\mathbb{N}^{-}}$least numbers not yet in the domain of our partial orders and uses them as the witnesses $q_{\alpha}^{0}$ and $q_{\alpha}^{1}$. In the $\leq_{0}$ order, it places them immediately above $u_{\alpha^{-}}^{0}$, incomparable with each other and incomparable with all nodes which are not below $u_{\alpha^{-}}^{0}$. In the $\leq_{1}$ order, it places them immediately above $u_{\alpha^{-}}^{1}$, incomparable with each other and incomparable with all nodes which are not below $u_{\alpha^{-}}^{1}$. Set $u_{\alpha}^{k}=q_{\alpha}^{k}$ and let $n_{\alpha}$ denote the number of points which are currently in the partial orders.

2. Wait for a stage $t$ such that $\varphi_{e}^{X_{i}}(x)$ appears to converge for all $x \leq_{\mathbb{N}} n_{\alpha}$. If $\varphi_{e}^{X_{i}}$ is not one-to-one on these points, then $\mathcal{R}_{\alpha}$ takes no further action at this stage. Otherwise, let $x$ be the $\leq_{\mathbb{N}^{-}}$least point $\leq_{\mathbb{N}} n_{\alpha}$ such that $\varphi_{e}^{X_{i}}(x)$ appears to converge to a point $y$ such that $q_{\alpha}^{j} \leq_{j} y$.

3. When this convergence occurs, initialize all lower priority requirements and set $u_{\alpha}^{j}=$ $y$. The point $y$ is $q_{k}^{\beta}$ for some lower priority strategy $\mathcal{R}_{\beta}$ and some $k \in\{0,1\}$. Set $u_{\alpha}^{1-j}=q_{1-k}^{\beta}$. If some computation for $\varphi_{e}^{X_{i}}(z)$ with $z \leq_{\mathbb{N}} n_{\alpha}$ changes, return to step 2 . Otherwise, the values of $u_{\alpha}^{0}$ and $u_{\alpha}^{1}$ remain fixed.

We sketch the verification that this construction succeeds. The construction is finite injury, so each requirement $\mathcal{R}_{\alpha}$ eventually stops being initialized and defines final values for $q_{\alpha}^{0}, q_{\alpha}^{1}$ and $n_{\alpha}$. After finitely many more stages, the computations $\varphi_{e}^{X_{i}}(z)$ for $z \leq_{\mathbb{N}} n_{\alpha}$ settle down and the values of $u_{\alpha}^{0}$ and $u_{\alpha}^{1}$ stabilize. At this point, $\mathcal{R}_{\alpha}$ is met exactly as in the informal description of $\mathcal{R}_{\langle 0,0,0\rangle}$. Therefore, both $\leq_{0}$ and $\leq_{1}$ satisfy wqo in $\mathcal{A}$.

Second, we check the antichain requirements. It is clear by induction on the stages of the construction that at all stages and for all triples $\beta=\langle e, i, j\rangle$, the points $u_{\beta}^{0}$ and $u_{\beta}^{1}$ are incomparable in both the $\leq_{0}$ and $\leq_{1}$ orders. Furthermore, in both $\leq_{0}$ and $\leq_{1}$, the cones above $u_{\beta}^{0}$ and $u_{\beta}^{1}$ are disjoint. For a contradiction, assume that there are points $x$ and $y$ such 
that $x<_{0} y$ and $x<_{1} y$. In this case, we must have $x<_{\mathbb{N}} y$ and hence $y$ is placed in the partial orders after $x$. Let $s$ be the stage at which $y$ is placed in the partial orders and let $\beta$ denote the triple such that $\mathcal{R}_{\beta}$ places $y$ in the partial orders. Since $x<_{0} y, \mathcal{R}_{\beta}$ must be forced to work in the cone above $x$ by a higher priority requirement. That is, there is a triple $\alpha$ such that $\mathcal{R}_{\alpha}$ has higher priority than $\mathcal{R}_{\beta}$ and $x=u_{\alpha}^{0}$ at stage $s$. However, in this situation, $\mathcal{R}_{\beta}$ is forced to make $u_{\alpha}^{1}<_{1} y$ and hence to make $x=u_{\alpha}^{0} \nless_{1} y$, giving us the desired contradiction.

Corollary 4.4. Let $P_{1}$ be any property from among wqo, wqo(ext) and wqo(anti). Let $P_{2}$ be any property from among wqo, wqo(set), wqo(ext) and wqo(anti). $\mathrm{WKL}_{0}$ is not strong enough to prove that if $\preceq_{0}$ and $\preceq_{1}$ satisfy $P_{1}$ then $\preceq_{0} \cap \preceq_{1}$ satisfies $P_{2}$.

Proof. Fix $P_{1}$ and $P_{2}$ from the lists of properties in the statement of the corollary and consider the partial orders $\leq_{0}$ and $\leq_{1}$ and the model $\mathcal{A}$ of $\mathrm{WKL}_{0}$ from Theorem 4.3. Since $\mathrm{RCA}_{0}$ suffices to prove wqo $\rightarrow$ wqo(ext) and wqo $\rightarrow$ wqo(anti), we have that $\leq_{0}$ and $\leq_{1}$ satisfy $P_{1}$ in $\mathcal{A}$. The fact that $\leq_{0} \cap \leq_{1}$ is an infinite antichain means that it does not satisfy $P_{2}$. (If $P_{2}$ is wqo(ext), then the linear order defined by $n \leq^{\prime} m$ if and only if $n \geq_{\mathbb{N}} m$ is a non well-founded linear extension of $\leq_{0} \cap \leq_{1}$.)

The following theorem allows us to transfer our results on intersection to products. The proof for the case when $P$ is the property wqo is contained in Lemma 5.20 of [7] and essentially the same proof works for each of the other properties $P$.

Lemma 4.5 (Marcone). Let $P$ be any of the properties wqo, wqo(set), wqo(ext) or wqo(anti). Over $\mathrm{RCA}_{0}$, the following statements are equivalent.

1. If $Q_{1}$ and $Q_{2}$ satisfy $P$, then $Q_{1} \times Q_{2}$ with the product order satisfies $P$.

2. If $Q$ satisfies $P$ with respect to $\preceq_{1}$ and $\preceq_{2}$, then $Q$ satisfies $P$ with respect to $\preceq_{1} \cap \preceq_{2}$.

Corollary 4.6. Let $P$ be any of the properties wqo, wqo(ext), wqo(anti) or wqo(set). $\mathrm{RCA}_{0}$ is strong enough to prove that if $\left(Q_{1}, \preceq_{1}\right)$ and $\left(Q_{2}, \preceq_{2}\right)$ satisfy wqo(set), then $Q_{1} \times Q_{2}$ under the product order satisfies $P$.

Corollary 4.7. Let $P_{1}$ be any property from among wqo, wqo(ext) and wqo(anti). Let $P_{2}$ be any property from among wqo, wqo(set), wqo(ext) and wqo(anti). $\mathrm{WKL}_{0}$ is not strong enough to prove that if $\left(Q_{1}, \preceq_{1}\right)$ and $\left(Q_{2}, \preceq_{2}\right)$ satisfy $P_{1}$ then $Q_{1} \times Q_{2}$ under the product order satisfies $P_{2}$.

\section{Equivalent definitions for better quasi-orders}

We turn our attention to the notion of a better quasi-order. In this section, all strings are finite increasing sequences in $[\mathbb{N}]^{<\omega}$. We think of each string as enumerating a finite set in strictly increasing order. Similarly, we equate each infinite set $X$ with the $\mathbb{N}$-sequence of the elements of $X$ in strictly increasing order. For strings $s$ and $t$, we continue to write $s \sqsubseteq t$ if $s$ is an initial segment of $t$, but we also write $s \subseteq t$ (or $s \subset t$ ) if the finite set represented by $s$ 
is a subset (or strict subset respectively) of the finite set represented by $t$. Similarly, we write $n \in s$ if $n$ is an element of the finite set represented by $s$.

Given a set $B \subseteq \mathbb{N}^{<\omega}$, we define base $(B)$ to be $\bigcup B$, or more formally

$$
\operatorname{base}(B)=\{n \mid \exists s \in B(n \in s)\} .
$$

It is easy to show that the existence of base $(B)$ for arbitrary $B$ is equivalent to $\mathrm{ACA}_{0}$ (see Lemma 1.4 in [7]). Therefore, when we make statements like "base $(B)$ is infinite" in a subsystem weaker than $\mathrm{ACA}_{0}$, we mean the formal statement $\forall m \exists n>m \exists s \in B(n \in s)$. Similarly, when we say $X$ is a subset of base $(B)$, we mean $\forall x \in X \exists s \in B(x \in s)$.

Definition 5.1. $\left(\mathrm{RCA}_{0}\right) B \subseteq[\mathbb{N}]^{<\omega}$ is a block if

1. $\operatorname{base}(B)$ is infinite;

2. $\forall X \in[\operatorname{base}(B)]^{\omega} \exists s \in B(s \sqsubset X)$;

3. $\forall s, t \in B(s \not \subset t)$.

$B$ is a barrier if it satisfies the first two conditions above and the (stronger) third condition that $\forall s, t \in B(s \not \subset t)$. Notice that every barrier is a block.

Definition 5.2. $\left(\mathrm{RCA}_{0}\right)$ Let $s$ and $t$ be strings. We write $s \triangleleft t$ if there is are strings $u, v$ such that $u=u(0) * v$ (that is, $v$ is obtained by deleting the first element of $u$ ), $s \sqsubseteq u$ and $t \sqsubseteq v$.

Definition 5.3. $\left(\mathrm{RCA}_{0}\right)$ A quasi-order $(Q, \preceq$ ) is a better quasi-order (or bqo) if for every barrier $B$ and every function $f: B \rightarrow Q$, there exist $s, t \in B$ such that $s \triangleleft t$ and $f(s) \preceq f(t)$.

Classically, there are a number of equivalent definitions for a bqo. First, we can restrict the definition by only considering barriers $B$ such that base $(B)=\mathbb{N}$. Lemma 1.6 in [7] shows that $\mathrm{RCA}_{0}$ suffices to prove that base $(B)$ exists for a barrier $B$. Therefore, in $\mathrm{RCA}_{0}$, the definition of a bqo in terms of barriers with base $\mathbb{N}$ is equivalent to the one given above.

Second, we can replace the barriers in the definition of a bqo by blocks and we can restrict the use of blocks to those blocks $B$ with base $(B)=\mathbb{N}$. Below, we prove that $\mathrm{RCA}_{0}$ suffices to prove that $\operatorname{base}(B)$ exists for any block $B$. This result shows that, over $\mathrm{RCA}_{0}$, it is equivalent to define a bqo in terms of blocks and in terms of blocks with base $\mathbb{N}$. Finally, we present a method for passing from blocks to barriers which was first used by Marcone in [6] and we prove that this method works in $\mathrm{WKL}_{0}$. Together, these results show that in $\mathrm{WKL}_{0}$ the definition of bqo in terms of barriers is equivalent to the definition of bqo in terms of blocks.

Third, one could consider the condition bqo(set), which is defined analogously to wqo(set). If $B$ is a barrier and $Y$ is an infinite subset of base $(B)$, then there is a unique barrier $B^{\prime} \subseteq B$ such that base $\left(B^{\prime}\right)=Y: B^{\prime}=\{s \in B \mid s \subset Y\}$. In this situation, we call $B^{\prime}$ a subbarrier of $B$. A quasi-order $(Q, \preceq)$ satisfies bqo(set) if for all barriers $B$ and all functions $f: B \rightarrow Q$, there exists a subbarrier $B^{\prime} \subseteq B$ such that for all $s, t \in B^{\prime}$ if $s \triangleleft t$, then $f(s) \preceq f(t)$. Clearly, $\mathrm{RCA}_{0} \vdash$ bqo(set) $\rightarrow$ bqo, and in Theorem 4.9 of [7], it is shown that the implication bqo $\rightarrow$ bqo(set) is equivalent (over $R_{C A}$ ) to $A T R_{0}$. $\quad\left(A_{T R}\right.$ is the subsystem formed by 
extending $\mathrm{ACA}_{0}$ to allow definitions by the transfinite recursion of arithmetic predicates over well orders. It is significantly stronger than any of the systems considered in this paper.)

In the following lemma, we state a property of blocks which will enable us to prove that the base of any block exists in $\mathrm{RCA}_{0}$. In the statement of the lemma, we do not want to assume that the base of the block $B$ exists. Therefore, the requirement that $s \subset$ base $(B)$ is shorthand for the formal statement that for all $n<|s|$, there is a $u \in B$ such that $s(n) \in u$.

Lemma 5.4. $\left(\mathrm{RCA}_{0}\right)$ If $B$ is a block, then there is a string $s$ and a number $k$ such that $s \subset \operatorname{base}(B), \forall u \sqsubseteq s(u \notin B)$, and $\forall t \in B(s \sqsubset t \rightarrow|t| \leq|s|+k)$.

Proof. For a contradiction, assume that there is no such string $s$. That is, if $s \subset \operatorname{base}(B)$ and for all $r \sqsubseteq s, r \notin B$, then it is not the case that there is a $k$ for which all $t \in B$ with $s \sqsubset t$ satisfy $|t| \leq|s|+k$. We define a set $Z \in[\operatorname{base}(B)]^{\omega}$ such that $Z$ has no initial segment $B$. This set contradicts the fact that $B$ is a block and completes the proof.

We begin to define $Z$ by applying our assumed hypothesis with $s$ equal to the empty string. Our assumption tells us that there must be an $s_{0} \in B$ with $\left|s_{0}\right| \geq 2$. Take the $<_{\mathbb{N}}$-least such string and let $n_{0}=s_{0}(0)$ be the least element of $Z$. We know that $\left\langle n_{0}\right\rangle \notin B$ (since $\left\langle n_{0}\right\rangle$ is a proper initial segment of $s_{0}$ ) so by our assumption there must be a string $s_{1} \in B$ with $s_{1}(0)=n_{0}$ and $\left|s_{1}\right| \geq 3$. Take the $<_{\mathbb{N}^{-}}$least such string and let $n_{1}=s_{1}(1)$ be the second smallest element of $Z$. Notice that neither $\left\langle n_{0}\right\rangle$ nor $\left\langle n_{0}, n_{1}\right\rangle$ is in $B$.

In general, assume that we have defined $n_{0}<n_{1}<\cdots<n_{k}$ to be an initial segment of $Z$ and that $\left\langle n_{0}, \cdots, n_{i}\right\rangle \notin B$ for all $i \leq k$. By our assumption, there is an element $s_{k+1} \in B$ with $\left\langle n_{0}, \cdots, n_{k}\right\rangle \sqsubset s_{k+1}$ and $\left|s_{k+1}\right| \geq k+3$. Fix the $<_{\mathbb{N}}$ least such string and let $n_{k+1}=s_{k+1}(k+1)$ be the next smallest element in $Z$. Notice that the induction hypothesis is maintained so the recursion can continue.

Formally, we have defined a function $f$ such that $f(k)=n_{k}$ and we let $Z$ be the range of $f$. $Z$ exists as a set since $k \in Z$ if and only if $\exists i \leq k\left(k=n_{i}\right)$. Also, by our construction, the nonempty initial segments of $Z$ are precisely $\left\langle n_{0}, \ldots, n_{q}\right\rangle$ for $q \in \mathbb{N}$ and none of these sequences is in $B$. This contradicts the fact that $B$ is a block and finishes the proof.

Lemma 5.5. $\left(\mathrm{RCA}_{0}\right)$ If $B \subset[\mathbb{N}]^{<\omega}$ is a block, then base $(B)$ is a set.

Proof. It suffices for us to give a $\Pi_{1}^{0}$ definition of the base of $B$. By Lemma 5.4, we can fix a string $s$ and a number $k$ such that $s \subset$ base $(B), \forall u \sqsubseteq s(u \notin B)$ and for all $t \in B$ such that $s \sqsubset t$ we have $|t| \leq|s|+k$. Let $n$ be the maximum value in $s$. We prove that base $(B)$ consists of a finite subset of $\{0, \ldots, n\}$ plus the set $X$ consisting of all $m>n$ for which

$\forall m_{1}<\cdots<m_{k-1}\left(\left[m<m_{1} \wedge\left(m_{1}, \ldots, m_{k-1} \in \operatorname{base}(B)\right)\right] \rightarrow \exists i<k\left(s *\left\langle m, m_{1}, \ldots, m_{i}\right\rangle \in B\right)\right)$.

Notice that the definition of $X$ is $\boldsymbol{\Pi}_{1}^{0}$ since the predicate " $\in$ base $(B)$ " is $\boldsymbol{\Sigma}_{1}^{0}$. The fact that $X \subseteq \operatorname{base}(B)$ is immediate from the definition of $X$. To see that $(\operatorname{base}(B) \backslash\{0, \ldots, n\}) \subseteq X$, fix $m_{1}, \ldots, m_{k-1} \in \operatorname{base}(B)$ such that $m<m_{1}<\cdots<m_{k-1}$ and let $Y$ be an infinite set such that $s *\left\langle m, m_{1}, \ldots, m_{k-1}\right\rangle \sqsubset Y$. Such a $Y$ does exist because base $(B)$ is infinite and hence $\mathrm{RCA}_{0}$ proves that it contains an infinite subset. $Y$ must have an initial segment in $B$ and we know that for all $r \sqsubseteq s, r \notin B$. Therefore, some extension of $t$ of $s$ with $|t| \leq|s|+k$ and $t \sqsubset Y$ must be in $B$. Therefore, there is an $i<k$ such that $s *\left\langle m, m_{1}, \ldots, m_{i}\right\rangle \in B$. 
In the previous paragraph, we defined $X$ using a $\Pi_{1}^{0}$ formula and then showed that $X$ is equal to the set of all $m>n$ such that $m \in$ base $(B)$. Therefore, $X$ also has a $\Sigma_{1}^{0}$ definition and hence it exists as a set. Furthermore, by bounded $\Sigma_{1}^{0}$ comprehension (which holds in RCA see [11, Theorem II.3.9]), the set $Z$ of all $m \leq n$ such that $m \in \operatorname{base}(B)$ exists. Therefore, $\operatorname{base}(B)=X \cup Z$ exists.

By Lemma 5.5, we can assume without loss of generality in $\mathrm{RCA}_{0}$ that our blocks have base $\mathbb{N}$. This assumption is convenient since it allows us to avoid having to repeatedly state that we only work with strings whose elements come from base $(B)$.

Definition 5.6. $\left(\mathrm{RCA}_{0}\right)$ For strings $s$ and $t$, we write $t \ll s$ if and only if $|t|=|s|$ and $\forall i<|t|(t(i) \leq s(i))$.

Given a block $B$ with base $\mathbb{N}$, we define a tree $T(B)$ such that $B$ is exactly the set of leaves of $T(B)$. We define a supertree $T^{*}(B)$ of $T(B)$ and let $B^{*}$ be the set of leaves of $T^{*}(B)$. The set $B^{*}$ will be a barrier. (A tree in this context means a nonempty subset of $[\mathbb{N}]^{<\omega}$ which is closed under initial segments and for which every string is strictly increasing.)

Definition 5.7. $\left(\mathrm{RCA}_{0}\right)$ For a block $B$ with base $\mathbb{N}$, we define

$$
\begin{gathered}
T(B)=\{s \mid \forall t \sqsubset s(t \notin B)\} \\
T^{*}(B)=\{t \mid \exists s \ll t(s \in T(B))\} \\
B^{*}=\left\{t \in T^{*}(B) \mid \forall u\left(t \sqsubset u \rightarrow u \notin T^{*}(B)\right)\right\} .
\end{gathered}
$$

Notice that $T(B)$ and $T^{*}(B)$ can be defined in $\mathrm{RCA}_{0}$ (because the quantifications $\forall t \sqsubset s$ and $\exists s \ll t$ are bounded, since $s \ll t$ implies $|s|=|t|)$. At first, it appears that $B^{*}$ requires $\mathrm{ACA}_{0}$ to be defined since our trees are infinitely branching. We show below that this is not the case, but first we state the following obvious lemma.

Lemma 5.8. $\left(\mathrm{RCA}_{0}\right) T(B) \subseteq T^{*}(B)$.

Proof. Obvious from the definition since $s \ll s$ for any string $s$.

We prove the following characterization of $B^{*}$ : for any $t \in T^{*}(B)$

$$
t \in B^{*} \Leftrightarrow \forall s \ll t(s \in T(B) \rightarrow s \in B) .
$$

This characterization shows that $\mathrm{RCA}_{0}$ is sufficient to define $B^{*}$. To see that $(\Rightarrow)$ holds, suppose there is an $s \ll t$ such that $s \in T(B)$ and $s \notin B$. Then, for some $n>s(|s|-1)$, the sequence $s *\langle n\rangle$ is in $T(B)$. Hence $t$ has infinitely many extensions in $T^{*}(B)$ and therefore is not in $B^{*}$. To see that $(\Leftarrow)$ holds, suppose that $t \notin B^{*}$ and fix $u \in T^{*}(B)$ such that $t \sqsubset u$. Because $u \in T^{*}(B)$, there is a $v$ such that $v \ll u$ and $v \in T(B)$. Let $s$ be $v$ restricted to $|t|$. Notice that $s$ is an element of $T(B), s \ll t$ and $s \sqsubset v$. Therefore, $s$ is not a leaf in $T(B)$ and hence is not an element of $B$. This establishes the equivalence.

Lemma 5.9. $\left(\mathrm{WKL}_{0}\right)$ If $T(B)$ has no infinite path, then $T^{*}(B)$ has no infinite path. 
Proof. Suppose that $T^{*}(B)$ has an infinite path $X=\left\{x_{0}<x_{1}<\cdots\right\}$. For each $n$, let $\sigma_{n}=\left\langle x_{0}, \ldots, x_{n-1}\right\rangle$. Since $\sigma_{n} \in T^{*}(B)$, there must be an element $s \in T(B)$ such that $s \ll \sigma_{n}$. Let $S=\left\{s \in T(B) \mid s \ll \sigma_{|s|}\right\}$. S is a finitely branching subtree of $T(B)$ and the branching is bounded at level $n$ by the function $f(n)=x_{n}$. Furthermore, since $X$ is a path through $T^{*}(B)$, $S$ is infinite. Applying Bounded König's Lemma (which is available in $\mathrm{WKL}_{0}$, see [11, Lemma IV.1.4]) to $S, S$ must have an infinite path (which is of course also a path in $T(B)$ ).

Restating Lemma 5.9 in purely computability theoretic language yields the following corollary.

Corollary 5.10. If $T(B)$ has no low path, then $T^{*}(B)$ has no computable path.

Proof. If $X$ is a computable path through $T^{*}(B)$, then the bounding function $f$ from the proof of Lemma 5.9 is computable. Hence, $S$ is a computably bounded $\Pi_{1}^{0}$ class and by the Low Basis Theorem must have a low path.

Lemma 5.11. $\left(\mathrm{WKL}_{0}\right)$ If $B$ is a block with base $\mathbb{N}$, then $B^{*}$ is a barrier.

Proof. We check the properties required of a barrier. First, notice that because $B$ is a block, every infinite set $Y$ has some initial segment $s \sqsubset Y$ with $s \in B$. Furthermore, $s$ cannot have any extension in $T(B)$. Therefore, $T(B)$ has no infinite path. By Lemma 5.9, $T^{*}(B)$ has no infinite path, which implies that every $Y$ has an initial segment $t \sqsubset Y$ with $t \in T^{*}(B)$. Therefore, $B^{*}$ contains initial segments of every set. Second, because $T^{*}(B)$ has no infinite paths, Lemma 5.8 shows that every $s \in B$ has some extension $t \sqsupseteq s$ such that $t$ is a leaf in $T^{*}(B)$. Therefore, base $(B) \subseteq$ base $\left(B^{*}\right)$, so base $\left(B^{*}\right)=\mathbb{N}$. Third, fix distinct leaves $s$ and $t$ in $T^{*}(B)$ and assume for a contradiction that $s \subset t$. Notice that $s \subset t$ implies that $|s|<|t|$ and if $|s|=n$ then $t \mid n \ll s$. Since $t \in T^{*}(B)$, there must be a $\sigma \in T(B)$ such that $|\sigma|=|t|$ and $\sigma \ll t$. Restricting to an initial segment gives $\sigma|n \ll t| n \ll s$. Hence, there is a string $u$ properly extending such that $|u|=|\sigma|$ and $\sigma \ll u$. Therefore, $u \in T^{*}(B)$. However, this contradicts the fact that $s$ is a leaf on $T^{*}(B)$.

Theorem 5.12. $\left(\mathrm{WKL}_{0}\right)$ A quasi-order $(Q, \preceq)$ is a bqo if and only if for every block $B$ with base $\mathbb{N}$ and every function $f: B \rightarrow Q$, there exist $s, s^{\prime} \in B$ such that $s \triangleleft s^{\prime}$ and $f(s) \preceq f\left(s^{\prime}\right)$.

Proof. The $(\Leftarrow)$ direction is clear since every barrier is a block. For the $(\Rightarrow)$ direction, let $(Q, \preceq)$ be a bqo and fix both a block $B$ and a function $f: B \rightarrow Q$. We have to find the corresponding $s$ and $s^{\prime}$. Let $B^{*}$ be the barrier associated to $B$ as above. Define $g: B^{*} \rightarrow B$ by setting $g(t)$ to be the unique $s \in B$ such that $s \sqsubseteq t$. (It is not hard to see that such $s$ must exist and be unique since $B$ is a block and $T(B) \subseteq T^{*}(B)$.) Define $f^{*}: B^{*} \rightarrow Q$ by composing $f$ and $g$. Since $Q$ is a bqo, there are $t, t^{\prime} \in B^{*}$ such that $t \triangleleft t^{\prime}$ and $f^{*}(t) \preceq f^{*}\left(t^{\prime}\right)$. Let $s=g(t)$ and $s^{\prime}=g\left(t^{\prime}\right)$. It is clear that $s, s^{\prime} \in B$ and $f(s)=f^{*}(t) \preceq f^{*}\left(t^{\prime}\right)=f\left(s^{\prime}\right)$. Because $t \triangleleft t^{\prime}$, we can fix strings $u$ and $v$ such that $u=u(0) * v, t \sqsubseteq u$ and $t^{\prime} \sqsubseteq v$. Since $s \sqsubseteq t$ and $s^{\prime} \sqsubseteq t^{\prime}$, the strings $u$ and $v$ also witness that $s \triangleleft s^{\prime}$. 


\section{References}

[1] Peter Cholak, Carl Jockusch \& Ted Slaman, The strength of Ramsey's Theorem for pairs, Journal of Symbolic Logic, 66(1) March 2001, 1-55.

[2] Rodney G. Downey, Computability theory and linear orderings, in Y.L. Ershov, S.S. Goncharov, A. Nerode \& J.B. Remmel editors, Handbook of Recursive Mathematics: Volume 2, 823-976, North Holland, Amsterdam, 1998.

[3] Rodney G. Downey, Denis R. Hirschfeldt, Steffen Lempp \& Reed Solomon, Computability-theoretic and proof-theoretic aspects of partial and linear orderings, to appear in Israel Journal of Mathematics.

[4] Eberhard Herrmann, Infinite chains and antichains in computable partial orderings, Journal of Symbolic Logic, 66(2) June 2001, 923-934.

[5] Denis R. Hirschfeldt \& Richard A. Shore, Some facts about linear orderings provable in $\mathrm{RT}_{2}^{2}$, to appear.

[6] Alberto Marcone, Foundations of bqo theory, Transactions of the American Mathematical Society, 345(2), October 1994, 641-660.

[7] Alberto Marcone, Wqo and bqo theory in subsystems of second order arithmetic, to appear in S.G. Simpson editor, Reverse mathematics 2001.

[8] Eric C. Milner, Basic wqo- and bqo-theory, in Ivan Rival, editor, Graphs and order, 487-502, D. Reidel, Dordrecht, 1985.

[9] Stephen G. Simpson, Bqo theory and Fraïssé's Conjecture, in R. Mansfield \& G. Weitkamp, Recursive aspects of descriptive set theory, 124-138, Oxford University Press, Oxford 1985.

[10] Stephen G. Simpson, Ordinal numbers and the Hilbert Basis Theorem, Journal of Symbolic Logic, 53(3) September 1988, 961-974.

[11] Stephen G. Simpson, Subsystems of second order arithmetic, Springer-Verlag, 1998. 